\title{
People and paradigms: mental networks and the ventromedial prefrontal cortex
}

Lorin Friesen, mentalsymmetry.com, Abbotsford, BC, Canada

\section{Abstract}

A cognitive meta-theory has been developed by looking for common patterns of behavior in different fields (Friesen, 2021c). A primary concept of this theory is the mental network (MN), which can be thought of as an emotionally imposed schema. The MN provides a unified explanation for the diverse functions of the ventral medial prefrontal cortex (vmPFC). Going further, MNs provide the building blocks for forming concepts of self and others, and Theory of Mind uses MNs to predict the thinking and behavior of others. Another primary concept of the meta-theory is the idea that theories generate emotions (Friesen, 2021b). Facts are not emotional, but when facts are assembled into theories, then an emotion will be generated based upon order-within-complexity. Putting these two concepts together leads to the idea of a theoretical mental network (TMN). The TMN provides a unified explanation for Thomas Kuhn's concept of paradigms and paradigm shifts, ideologies, sweeping statements, and conspiracy theories, as well as explaining many facets of religion including mysticism, theology, fundamentalism, and associated concepts of God.

\section{Introduction}

This paper is divided into two parts. The first part will describe the mental network (MN) and show that it provides an explanation for the varied functions of the vmPFC. The purpose of this first part is to provide a detailed description of the $\mathrm{MN}$ and to place this description upon a solid foundation of empirical evidence. This means looking at technical details and quoting extensively from neurological papers. The second part will introduce the concept of theoretical emotion and then combine this with the mental network. The resulting concept of a theoretical mental network (TMN) will then be used to explain how theories affect human thought and behavior. The first part contains more neurological details than the typical paper on psychology, while the second part contains more psychological references than the typical neurological paper. This division is necessary because there is enough neurological evidence to suggest that the role which the vmPFC plays with normal MNs also extends to TMNs but not enough neurological evidence to describe this role in detail. Therefore, one must turn to other sources to fill in the gaps. And these other sources make it possible to discuss TMNs from a larger perspective than the limited viewpoint of the typical neurological experiment.

A wide range of functions have been ascribed to the vmPFC including value (Bartra, 2013), self (Stendardi, 2021), schemas (Gilboa, 2017), social interaction (Bzdok, 2013), morality (Cameron, 2018), and addiction (Konova, 2019). A cognitive model that maps in detail onto neurology (Friesen, 2019) has been developed. The methodology used to develop this model was described in an article (Friesen, 2021c) which also referred to the concept of mental networks. The first section of this paper proposes that mental networks (MNs) provide an explanation for the diverse functions of the vmPFC. 
An $\mathrm{MN}$ is a collection of emotional memories that have coalesced to function as a unit. It may form through repetition or in response to some emotional incident. Triggering one memory in an MN will activate the entire network, which will then exert emotional pressure to impose its structure upon thought and/or behavior. If an MN succeeds in imposing its structure upon the mind, then it will function invisibly, providing a subtle emotional pressure to maintain the status quo. But if an $\mathrm{MN}$ is triggered and continues to encounter data incompatible with its structure, then it will generate a strong negative emotion. Providing the MN with compatible input will remove this emotional discomfort, while continuing the discrepancy will increase the emotional discomfort until the MN eventually falls apart and its content functions again as disconnected memories. Putting this all together, a mental network can be viewed as an emotionally imposed schema.

\section{MNs and vmPFC Function}

The various attributes of a MN can be seen in neurological papers that describe the functioning of the vmPFC. ${ }^{1}$ Looking first at the general idea of an emotionally imposed schema, Gilboa (2017) defines a schema as an "active knowledge structure" (p. 619) that is "determined by the accumulation of subjective experiences” (p. 629). "The vmPFC is thought to activate relevant, and suppress irrelevant, schematic information" (p. 625). This describes a collection of experiential memories that is functioning as a unit and is imposing its structure upon the mind. This activation "assesses the subjective relevance and value of new information to the current context, a function that occurs rapidly and automatically” (p. 628). Thus, a new situation will trigger an existing MN which will then exert emotional pressure to impose its structure. Some research on the vmPFC focuses more upon the content of a MN. For instance, Spalding (2018) summarizes that "the vmPFC plays a role in schema formation, consolidation, and retrieval” (p. 3773). Other research on the vmPFC emphasizes the emotional side. For example, Lin (2016) suggests that the "vmPFC plays a role in the generation of affective meaning, in the association of events with emotional responses” (p. 8).

Triggering an MN will retrieve a context with the associated feelings. Benoit (2014) suggests "a critical role for the ventromedial prefrontal cortex in simulating what it may feel like to experience... future events” (p. 16550). Notice that an $\mathrm{MN}$ is being internally triggered. One is thinking about some situation, this is activating an $\mathrm{MN}$, and the $\mathrm{MN}$ is imposing its structure upon the mind along with the associated feelings. However, this $\mathrm{MN}$ was initially formed out of emotional experiences that were repeated, because "activation in the vmPFC was greater during the imagination of combinations of more familiar people and places" (p. 16551). Similarly, Schacter (2017) summarizes that "the contribution of the vmPFC to episodic simulation may be twofold: accessing schematic knowledge and processing anticipated affect” (p. 47).

A triggered $\mathrm{MN}$ will create the assumption that existing patterns will be repeated. Thus, "vmPFC recruitment leads to better memory for knowledge-congruent information but also to a heightened susceptibility to commit knowledge-congruent memory errors” (Brod, 2019, p. 1326). A 'knowledge-

1 Neurological papers use inconsistent capitalization when referring to this region, ranging from vmpfc to VMPFC. I will be using the form vmPFC but will preserve the form used by the author when making a direct quote. 
congruent memory error' is when one mistakenly assumes that a previous pattern will be repeated. This affects verbal interaction, because “listeners' ventromedial prefrontal cortex encodes the probabilistic inference of what a cooperative speaker should say given a communicative goal and context, even when such inferences are irrelevant for reference resolution” (Brod, 2018, p. 8). It also impacts social interaction, because "the vmPFC is involved in calibrating social actions by processing implied, rather than explicit, social information” (Mi, 2021, p. 9). Notice that an MN is being triggered by partial information and is responding by automatically imposing its entire structure upon the mind, whether this is required or not.

When an $\mathrm{MN}$ is triggered, then this will lead to the feeling that the content of that $\mathrm{MN}$ is correct. Hebscher (2016) suggests that "The vmPFC is thought to mediate an earlier, more automatic, intuitive 'feeling of rightness"” (p. 47). Similarly, Gherman (2018) concludes that "activity in only one area reliably indicated how confident the volunteers felt before they had made their choice... the ventromedial prefrontal cortex" (p. 47). This leads to emotionally motivated decisions based upon which MNs are triggered by the environment. "The vmPFC drives our decisions and triggers emotional states by shifting decisions based on situational factors, such as cognitive demand and emotional salience” (Manuel, 2019, p. 6).

MNs will naturally cause cultural groups to emerge. People with similar emotional experiences will develop similar MNs and it will 'feel right' when others are motivated by their MNs to behave in a manner that is consistent with my personal MNs. Similarly, it will 'feel wrong' when others are motivated by their MNs to behave in a manner that is inconsistent with my MNs. "Being imitated compared to not being imitated activates brain areas that have been associated with emotion and reward processing, namely medial orbitofrontal cortex/ ventromedial prefrontal cortex” (Kühn, 2010, p. 384). And distinguishing others who are similar from those who are dissimilar activates regions with the mPFC (Mitchell, 2006).

An MN does not generate mental content. Instead, it packages mental content into schema that are emotionally imposed upon thought and behavior. This emotional imposition requires the vmPFC. On the one hand, in "patients with vmPFC damage [there is] a stark dissociation between these patients' ability to reason abstractly about social decisions and their ability to act upon this reasoning in a congruent manner” (Bowren, 2018, p. 281). On the other hand, vmPFC activity is greater in those who are 'willing to fight and die' for their culture or beliefs (Pretus, 2019). Looking at this from a different perspective, moral judgments can be interpreted as MNs of expected behavior imposing themselves upon the mind. On the one hand, "Patients with vmPFC damage had weaker implicit moral evaluations of moral transgressions" (Cameron, 2018, p. 267). On the other hand, there is "VMPFC activity in response to targets of higher moral status” (Cloutier, 2014, p. 539).

An MN will also impose its structure upon the mind when it is triggered during conversation. “Listeners' ventromedial prefrontal cortex encodes the probabilistic inference of what a cooperative speaker should say given a communicative goal and context, even when such inferences are irrelevant” (Mi, 2021, p. 1). This happens automatically even when it is not required. "The pragmatic likelihood estimate was encoded in the listener vmPFC even when not required for decoding the intended 
referents” (p. 5). As the author suggests, this provides an explanation for Grice's classic principles of implicature (Grice, 1975). However, a listener is not usually choosing consciously to follow rational principles - as Grice initially suggested. Instead, mental networks are being triggered by either the context or by certain words in the conversation, and these MNs are adding an implied meaning to the conversation by emotionally imposing their structure upon the mind. For instance, a single knowing glance from a friend or family member may be sufficient to bring to mind an entire network of likes and dislikes.

Addiction can also be interpreted as some MN emotionally imposing its structure upon thought and behavior. An MN generates strong pressure when it is triggered and not satisfied. Similarly, "The capacity of drug-associated cues to elicit indices of drug-craving intensifies or incubates with the passage of time during drug abstinence. This [is] associated with a time-dependent deregulation of ventromedial prefrontal cortex (vmPFC) function” (Miller, 2017, p. 629). A 'drug-associated cue' is some item or situation that triggers an MN of addiction. 'Drug-craving' describes the response of an MN when it is triggered but not satisfied. If an MN continues to be triggered and not satisfied, then it will eventually fall apart. This is referred to as extinction. And "The same VMPFC region reported to be engaged during the extinction of fear also supports extinction of drug cue and appetitive associations” (Konova, 2019, p. 412).

Extinguishing an MN needs to be distinguished from avoiding an MN. Extinction causes an MN to fall apart by repeatedly triggering it without satisfying it. Avoidance stops an MN from imposing its structure by preventing it from being triggered. This distinction can be seen in a study carried out of American Vietnam war veterans. "Narcotic use and narcotic addiction were extremely common in Vietnam... those with a history of deviant behaviour before Vietnam were particularly at risk; addiction was rare and brief after return, even when men continued to use narcotics; veterans re-addicted and entering treatment had as high a relapse rate as civilians” (Robins, 1993). Summarizing, fighting in Vietnam was so different than living as a civilian in America that MNs of addiction acquired in Vietnam ceased to be triggered when returning to America. That is an example of avoidance. However, this was not the case for individuals who acquired MNs of addiction within America. Instead, such individuals had to go through programs of drug treatment which attempted to extinguish MNs of drug addiction.

When more than one mental network is triggered at the same time, then each will exert emotional pressure to impose its structure upon the other. This leads over time to an emotional hierarchy of mental networks, with core mental networks imposing their structure upon lesser mental networks. Consistent with this, a meta-analysis found that vmPFC activity depends upon relative social status, noting "consistent involvement of the ventral striatum and ventromedial prefrontal cortex in downward comparison and consistent involvement of the anterior insula and dorsal anterior cingulate cortex in upward comparison” (Luo, 2018, p. 440). The anterior cingulate cortex performs cost/benefit calculations for goal-oriented behavior (Kolling, 2016.) This suggests that lower status leads to a drive for self-improvement while higher status emphasizes the existing emotional social context. However, lower social status does not always motivate self-improvement. For instance, a study of adolescent girls 
found that "when participants had relatively high vmPFC activation in response to a negative item, such as 'lonely' or 'anxious', they were more likely to endorse this item as self-descriptive compared to when they showed lower vmPFC activation.” (Barendse, 2020, p. 2). This is an example of an MN imposing the structure of an unpleasant social description upon the mind.

\section{The Formation and Study of MNs}

An MN can form immediately as a result of a traumatic incident. Using neurological language, “The vmPFC may act as a hub brain system that integrates sensory, affective, memory-related, and social information from diverse brain systems to coordinate behavioral and peripheral physiological responses according to contextual demands, particularly those demands that are appraised as psychologically stressful” (Ginty, 2019, p. 1). Notice that the vmPFC is responding to stress by imposing some pattern of response, consistent with the formation of an MN. A study of Vietnam War veterans "found a significantly reduced occurrence of PTSD [post-traumatic stress disorder] among those individuals with damage to one of two regions of the brain: the vmPFC and an anterior temporal area that included the amygdala. These results suggest that vmPFC and amygdala are critically involved in the pathogenesis of PTSD” (Koenigs, 2008, p. 232). The authors explained this by suggesting that the "vmPFC is critical for the re-activation of emotional states associated with past experiences" (p. 236), consistent with the concept of forming an $\mathrm{MN}$.

An MN may also form naturally after some time. "We found that remote memories were particularly well represented in ventromedial prefrontal cortex (vmPFC) compared to recent memories... Direct comparison of the two week old memories with themselves two years later confirmed that their representation within vmPFC had become more evident.” (Bonnici, 2018, p. 159). For instance, some cultural MNs form based upon the response of some group of people to a crisis situation, while other cultural MNs form as a result of some initially random behavior being repeated over time.

The formation of MNs after some time has been observed in the development of habits in rats. "It was not until the animals had been trained for a long time and the habit became fixed that the infralimbic activity changed. Strikingly, when it did, a chunking pattern then developed there, too. It was as though the infralimbic cortex was the wise one, waiting until the striatal evaluation system had fully decided that the behavior was a keeper before committing the larger brain to it” (Graybiel, 2014, pp. 42-43). The infralimbic cortex is part of the rat equivalent to the human vmPFC. Notice that the infralimbic cortex is committing to some sequence of actions after it has been repeated for a certain length of time. Notice also that the sequence of actions is being 'chunked' and treated as a single unit, consistent with the idea of a mental network imposing its entire structure upon the mind when it is triggered.

A mental network was described at the beginning of this paper as an emotionally imposed schema. And we have seen that both schema and emotional imposition can be found in research on the vmPFC. However, "The contribution of the vmPFC to contextual/spatial processing has been poorly integrated with the contribution of the vmPFC to fear. This may be attributed to the lack of communication between neuroscience subfields, and furthermore, the predominant use of discrete cues (mostly tones) in studies of the vmPFC's contribution to fear" (Pennington, 2017, p. 403). In other words, the idea of 
an 'emotionally imposed schema' may sound strange even though it is consistent with the function of the vmPFC. That is because those who are studying schema and those who are studying emotional imposition are not interacting with each other, and also because those who are studying emotional imposition are not using schema in their experiments. Looking at this more closely, Pennington found that "At no time during experimental testing did animals with lesions of the vmPFC display evidence of heightened fear responses. This runs in stark contrast to several reports that activation of the vmPFC is able to reduce anxiety-like behaviors, whereas inhibition produces the opposite response” (p. 404). Thus, the vmPFC does not just 'inhibit fear'. Instead, "The vmPFC contributes to contextual processing in fear learning” (p. 404) which is consistent with the idea of an emotionally imposed schema. Summarizing, it is too simplistic to say that the vmPFC inhibits emotional responses. A more accurate description is that the vmPFC imposes a pattern upon emotional responses.

Similarly, a study that looked at suicidal individuals found that "vmPFC-frontoparietal connectivity in suicide attempters reflects abnormal encoding of reinforcement... People who attempt suicide display intact connectivity, in contrast to non-suicidal people high in impulsivity. However, as shown by its impact on behavior, the information communicated by this intact connectivity is abnormal” (Brown, 2020, p. 1039). In other words, an MN usually performs the function of imposing structure upon impulsive behavior. But in suicidal individuals, MNs are using emotional pressure to impose destructive behavior upon the mind. In both cases, structure is being imposed emotionally upon the mind.

In the same way that the study of fear and the vmPFC has tended to use 'single tones' rather than schema, so neuroeconomics has tried to reduce the role of the vmPFC to the 'single tone' of some global economic value. "A major hope in neuroeconomics is to identify a single scalar value signal. This signal should be one that transcends multiple dimensions along which options can vary, and that can encode values of categorically different types of options. Finding the neural location of this hypothesized signal has been a major goal of neuroeconomics; a large amount of recent work has narrowed in on the vmPFC. However, other research casts doubt on the existence of a pure value signal” (Mehta, 2019, p. 5348). Instead, "We conclude that vmPFC plays a rich role in encoding and integrating multiple foraging-related variables during economic decisions” (p. 5336). In other words, the vmPFC establishes a context for economic choice.

Looking at this more carefully, the orbitofrontal cortex calculates subjective value which guides economic choice (Padoa-Schioppa, 2017). However, the subjective value generated by orbitofrontal neurons depends upon the context—which is determined by the hippocampus (Farovik, 2015). Strictly speaking, "Lesion studies indicate that OFC and the amygdala are the only regions strictly necessary for goal-directed behavior" (p. 739). Consistent with this, a study of patients with vmPFC damage found that the individual with bilateral vmPFC damage used "A more rational betting strategy than in healthy people” (Manohar, 2021, p. 33). This implies that the economic definition of value being used by neuroeconomics is a simplified interpretation of value which describes what the orbitofrontal cortex generates within some context when not being modified by MNs from the vmPFC. 
But the Nobel prize winning research of Tversky \& Kahneman (Tversky, 1992)—and behavioral economics in general-show that economic value in the average person is more than the simplistic concept of value taught in most economic classes. Instead, "Results suggest that vmPFC normally imposes contextual biases, which in healthy people may actually be suboptimal in some situations" (Manohar, 2021, p. 24). In the words of another author, "The vmPFC appears to be important for the context-appropriate retrieval of fear extinction memory” (Gonzalez, 2020, p. 462). Putting this together, the vmPFC provides the emotional framework for an MN while the actual content-and context - is being provided by other brain regions including the hippocampus (McCormick, 2018). In other words, "The vmPFC appears to work in concert with the BLA [basolateral amygdala] and hippocampus to guide the context-appropriate expression of fear” (Gonzalez, 2020, p. 463).

Summarizing, MNs are different than basic emotions. The orbitofrontal cortex assigns emotional labels to experiential memories (Rolls, 2020). An MN adds an additional emotion based upon congruence with some context. Consistent with this, a recent lesion study found that "Patients with VMF [ventromedial frontal] damage were impaired in making choices when value was uniquely predicted by the configuration of attributes, but intact when choosing based on elemental attribute values", concluding that “This region may be key for judging the emergent 'value of the forest,' rather than for integrating the individual 'value of each tree”” (Pelletier, 2019, p. 4124).

Finally, it should also be clarified that MNs do not directly generate motivation. Motivation is provided by dopamine and the ventral striatum, and the 'wanting' of motivation is different than the 'liking' of emotion (Berridge, 2016). Thus, when this paper talks about mental networks 'motivating behavior', this actually means that mental networks are generating emotions and these emotions are resulting in motivation from the ventral striatum through the involvement of dopamine.

\section{MNs and Self}

Turning now to the larger picture, a recent meta-study concluded "that social processes are functionally linked to DMPFC (dorsal mPFC), self processes are linked to AMPFC (anterior mPFC), and affective processes are linked to AMPFC and VMPFC” (Lieberman, 2019, p. 311). This suggests a hierarchy in which the orbitofrontal cortex assigns emotional labels to experiences, the vmPFC assembles these into MNs, and MNs form the building blocks for self and Theory of Mind.

Looking at this more carefully, MNs are composed of many kinds of emotional experiences which may or may not involve self. Self can be defined as the set of MNs that continually come to mind. MNs may be repeatedly triggered by physical sensation (via the insula), and knowledge and skill can also bring MNs repeatedly to mind (via the dorsolateral prefrontal) (Sui, 2017). The end result is that self becomes limited to the subset of MNs that are consistent with personal physical attributes, knowledge, and skills. For instance, an MN of 'flying through the air' may exist within a person's mind, but this $\mathrm{MN}$ is not a part of self because a physical body is incapable of flight. However, acquiring the skill of flying an airplane will cause an MN of 'flying through the air' to be regarded as an aspect of self. In a similar manner, familiar people will also become defined by the MNs that repeatedly come to mind when encountering these people. 
In most situations, factual knowledge about a situation will be used to determine which MNs are appropriate to model self or others. On the one hand, "the same networks involved in personal decision making are involved in making predictions about other people” (Kolling, 2021, p. 14). Thus, MNs make no inherent distinction between self and others. But on the other hand, the "ventromedial prefrontal cortex tracked the state of the world independent of the type of decision (personal, social), whereas dorsomedial regions adjusted their frame of reference to the use of privileged or shared information" (p. 2). Summarizing, MNs reside within the vmPFC while the dmPFC (and presumably amPFC) determines which of these MNs apply to some individual (including self) and which do not.

Identification happens when MNs that do not represent self are allowed to mingle with MNs that do represent self. "Identification involves a loss of one's sense of self as one in essence 'becomes' the character during the narrative experience" (Broom, 2021, p. 542). And "individuals higher in trait identification exhibit greater self-other neural overlap with fictional characters in the vmPFC" (p. 547). In this case, becoming immersed in some book or movie causes the MN that represents a fictional character to be repeatedly triggered, leading to the temporary feeling that self is that character, shown neurologically by an overlap in the vmPFC activity being generated by self and that character.

It is also possible to interact with imaginary characters in a parasocial manner. "A key difference between parasocial processes and identification is that one retains his/her sense of self during parasocial processes, imagining what it would be like to interact with or be friends with the character in question” (Broom, 2021, p. 548). In this case, the MN that represents some other person is being repeatedly triggered but the distinction between self and that other person is still being maintained. Note that social interaction with imaginary people is cognitively similar to interaction with real people, because in both cases the interaction is being guided by MNs that represent people.

Looking more closely at the relationship between MNs and concepts of self and others, an MN functions like a living being: It can be present or absent; it has likes and dislikes; it wants to be fed; it complains if it is not fed; it dies if it is starved. Thus, it is natural to use MNs to represent self and others. Interacting with a friend or family member will bring to mind the MN representing that person, which will impose memories of that person's likes and dislikes upon the mind. This explains why it feels as if a spouse or parent is 'living within my head'. It is disturbing when a person behaves inconsistently with the $\mathrm{MN}$ that represents that person. And remembering a dead person is an attempt to 'keep alive' the MN which represents that person. Thus, even when one is interacting socially with a real person and not an imaginary person, this interaction is still being emotionally influenced by the MN which represents that person and this MN will itself behave like a simplified version of that person.

The connection between people and the MNs that represent people is a fundamental concept in attachment theory, which refers to the MNs that represent parents as internal working models. "Experiences with parental sensitivity during distress... become internalized into Internal Working Models (IWMs). In attachment theory, IWMs are cognitive representations of these caregiving experiences that guide future interpersonal behavior and intimate relationships" (Bosmans, 2020, p. 287). Parents are represented by significant MNs within the mind of a child because of the extensive 
emotional interaction between a child and parents. As attachment theory points out, many of these childhood MNs are formed by traumatic situations and they will shape other MNs of social interaction.

\section{MNs and Naïve Thought}

Looking at this more generally, it is possible to explain many of the characteristics of Piaget's preoperational stage (McLeod, 2018) as thinking that is being shaped by childhood MNs. Quoting from the online article by McLeod, symbolic representation is "The ability to make one thing - a word or an object - stand for something other than itself." This happens naturally when some object triggers an $\mathrm{MN}$ that represents an object or person. With pretend play, "Toddlers often pretend to be people they are not (e.g. superheroes, policeman), and may play these roles with props that symbolize real life objects. Children may also invent an imaginary playmate.” In other words, objects are being used to trigger mental networks, and self is identifying with the MN that is currently active. Animism "is the belief that inanimate objects have human feelings and intentions." MNs, which act like living beings, are being used to interpret physical reality. Centration "is the tendency to focus on only one aspect of a situation at one time.” This happens when the currently active MN succeeds in imposing its structure upon the mind. Going further, artificialism "is the belief that certain aspects of the environment are manufactured by people (e.g. clouds in the sky)." Thus, the ability of MNs to impose their structure upon the mind is being regarded as a property of the physical world. Egocentrism "refers to the child's inability to see a situation from another person's point of view." This means that no other MNs influence the MN that is currently active. This expresses itself as play in which "Each child is absorbed in its own private world and speech is egocentric." Summarizing, MNs are dominant within the mind of the preoperational child, but they have not yet been organized into concepts of self and others or placed within a structure of Theory of Mind.

MNs also provide an explanation for the pre-scientific concept of impetus. A study that examined how students intuitively interpret physical movement found that "The intuitive theory bears a striking resemblance to the pre-Newtonian theory of impetus” (McCloskey, 1983, p. 123). Impetus views physical movement as a form of social interaction in which MNs struggle for dominance and form an emotional hierarchy with stronger MNs imposing their structure upon weaker MNs.

This can be seen, for instance, in how the path of a cannonball through the air is interpreted. "As the cannonball comes up, the force from the cannon is dissipating and the force of gravity is taking over. So it's slowing down. At the peak the force of the cannon and the force of gravity are fairly equal. That's the changeover point... As it makes the arc and begins to come down, gravity is overcoming the force from the cannon" (McCloskey, 1983, p. 126). Notice how the cannonball is being treated as a sort of MN that is imposing its behavior upon the environment. Gravity is also being treated as a kind of MN. When these two MNs interact with one another, then the MN of gravity ultimately prevails and imposes its structure upon the MN of the cannonball.

Looking at the example of an object dropped from a flying airplane, "An object acquires impetus when it is pushed or thrown but not when it is passively carried. Therefore, according to the impetus theory, when a carried object is dropped, there is no force to cause it to move forward, and so it falls straight 
down” (McCloskey, 1983, p. 127). In this case the MN of the carrier is imposing its structure upon the MNs of all the objects that it is carrying. Saying this another way, a carried object does not have the 'impetus' of its own MN but rather is being controlled by the MN of the carrier. Therefore, when a carried object is dropped, then its behavior is immediately determined by the MN of gravity because its own MN does not have any 'impetus'. Socially speaking, the carried object is merely a servant that has acquired a new master.

\section{MNs and Theory of Mind}

We have examined how MNs within the vmPFC provide the building blocks for forming concepts of self and others in the amPFC. Going further, MNs also play a fundamental role in Theory of Mind (ToM). Stated simply, social situations trigger MNs that represent people and situations, and these MNs will impose collections of likes and dislikes upon the mind. Thus, an MN provides a simple way for one person to predict what is happening within the mind of another. Because emotional experiences involving my physical body are a major source of MNs, ToM will naturally be biased by personal experience. However, it is possible to broaden the perspective of ToM through emotional experiences involving other people and other cultures. Consistent with this suggestion, research indicates that the basic functioning of ToM is independent of culture, but the development of various aspects of ToM is influenced by culture (Kuntoro, 2013).

ToM can be divided into an affective component that focuses upon predicting emotions and a cognitive component that predicts content. "While affective ToM was mostly impaired by VM [ventromedial frontal] damage, cognitive ToM was mostly impaired by extensive prefrontal damage, suggesting that cognitive and affective mentalizing abilities are partly dissociable” (Shamay-Tsoory, 2007, p. 3054). This combination of emotions and content reflects the nature of an MN, which uses emotions to impose content. Notice that affective ToM is primarily triggering MNs within the vmPFC, whereas cognitive ToM is using other regions within the prefrontal cortex to form a composite model of what the other person is thinking. A more recent study found that "belief-related effects were observed in both the DMPFC and VMPFC, whereas emotion-related effects were associated with VMPFC only" (CorradiDell'Acqua, 2014, p. 1183). This suggests that affective ToM is triggering MNs, whereas cognitive ToM is combining various MNs to come up with a more accurate model.

It was suggested that the characteristics of Piaget's precognitive stage can be explained as MNs controlling the mind. This dominance of childhood MNs can be seen neurologically. "The ventromedial prefrontal cortex (vmPFC) showed significantly stronger activation in adolescents in comparison to adults in the affective ToM condition. Current results indicate that the vmPFC might be involved in the development of affective ToM” (Vetter, 2014, p. 1022). In other words, MNs play a dominant role in the preschool child. But as the mind develops, these MNs within the vmPFC become organized by the dmPFC (assisted by other brain regions), making it possible to mix and match between various MNs, resulting in a Theory of Mind that is capable of predicting what another person is thinking.

ToM works most accurately with close friends. In fact, "A person's close friends and family know that person surprisingly well and can tell things about that person that he or she did not know before" 
(Vazire, 2008, p. 1214). That is because the repeated emotional experiences of friendship cause extensive MNs to form that represent the behavior of these friends, and because emotional experiences that are shared with friends will cause similar MNs to form within each person's mind.

When interacting with strangers, behavioral clues will trigger certain MNs, leading to initial impressions. Adding factual information allows ToM to go beyond these initial impressions to form composite images of people and situations. Looking at this in more detail, a study that introduced strangers to each other for the purpose of going on a date found that "The vmPFC automatically tracked the impression of faces... [which] indicates that the vmPFC is a neural locus of this affective gut reaction to facial stimuli" (Ito, 2020, p. 3054). However, "Reflected impression ratings formed through observation of the faces of others based on affiliation motivation are generally not accurate and do not accurately reflect the actual impression of others... [but] the accuracy of the reflected impression was improved by employing information acquired though the short social interaction” (p. 3055). Thus, MNs within the vmPFC led to an initial impression which was then modified by acquiring factual information.

We have looked at how MNs cause a person to jump to conclusions based upon initial impressions and we have seen that education can help ToM to become more accurate. Educated ToM has a tendency to jump to conclusions in a different manner. This can be seen in the way that researchers study ToM. "Most published articles on infant ToM have argued for a relatively sophisticated understanding of mental states in infants and I think there are several reasons for this. First, we know that at some point in human development we do come to understand mental states so it seems plausible that it might be as early as infancy. Second, and perhaps most important, mentalizing is so automatic for us as adults that it can be very difficult to understand how else infants could pass a ToM task. Yet, our adult view is utterly contaminated by our own skill at mentalizing and there is no guarantee that infants think like we do" (Ruffman, 2014, p. 268). Summarizing, adult ToM is reinterpreting the MNs of childish ToM, as illustrated by the previous example of going beyond initial impressions. But this reinterpretation becomes a handicap when attempting to study childish ToM. That is because this educated reinterpretation has becomes so natural that adult researchers who are studying childish ToM have a tendency to use their own adult ToM to reinterpret the childish MNs that they are observing.

This is apparent, for instance, in a paper on ToM entitled The scientist as child (Gopnik, 1996). The author recognizes that "As we deal with younger and younger children, it becomes more difficult to demonstrate that their understanding is genuinely theoretical" (p. 510). But despite admitting that this is a controversial opinion, the author concludes "Well, yes, actually, I do think it is theories all the way down" (p. 510). Notice how childish ToM is being interpreted through the lens of educated, adult theorizing.

The hypothesis of this paper is that ToM is based upon an underlying foundation of MNs. We have seen that naïve MNs are dominant within the mind of the pre-operational child. But we have just seen that something else based in theory can become dominant within the mind of an educated researcher to the extent of overwriting the very concept of naïve childish MNs. The rest of this paper will explore this possibility, suggesting that mental networks can form in the adult mind that are related to theories. The 
rationale for referring to 'the adult mind' can be found in Piaget's formal operational stage. "The formal operational stage begins at approximately age twelve and lasts into adulthood. As adolescents enter this stage, they gain the ability to think in an abstract manner by manipulating ideas in their head, without any dependence on concrete manipulation” (McLeod, 2010). I am not suggesting that the young child is incapable of theorizing. Instead, what is being examined here is the ability to use theories 'without any dependence on concrete manipulation'. Stated another way, a trained researcher is mentally able to 'think it is theories all the way down' because a trained researcher is functioning at the level of Piaget's formal operational stage. But the preoperational child is incapable of functioning cognitively at this level of abstraction. Therefore, childish ToM cannot be 'theories all the way down'. Instead, theories have to rest upon some underlying foundation of concrete experience.

So far, this paper has quoted extensively from neurological research on the vmPFC. The rest of this paper will examine the relationship between MNs and theories. This will include some references to the vmPFC, but it is difficult to find papers that examine the relationship between abstract thought and vmPFC activity. Thus, most of the evidence will come from a variety of fields that describe what happens when mental networks form that are related to theories.

\section{Theoretical Emotion and the TMN}

It has been suggested in another article that theories generate emotions (Friesen, 2021b). Stated in more detail, facts are not emotional. But when facts are assembled to form a theory, then this theory will generate a feeling of order-within-complexity. This feeling, which will be referred to as theoretical emotion, has historically been associated with beauty. And beauty has often been defined as a form of order-within-complexity. Symmetry, elegance, simplicity, grace, beauty, efficiency, harmony, fractals, and synchronized movement are all examples of order-within-complexity. Defining beauty as orderwithin-complexity is an ancient idea. For instance, the philosopher Plotinus stated, "Where the IdealForm has entered, it has grouped and coordinated what from a diversity of parts was to become a unity: it has rallied confusion into co-operation: it has made the sum one harmonious coherence: for the Idea is a unity and what it moulds must come into unity as far as multiplicity may” (Plotinus, 22 [Ennead I, 6]).

Theoretical emotion usually exists in combination with normal emotion. But theoretical emotion can be seen in pure form in mathematics. That is because mathematics uses words and symbols to construct abstract systems of order-within-complexity that are unrelated to the emotional experiences of normal life. And entire books have been written about the presence of beauty within mathematics (Montano, 2013).

Neurologically speaking, various forms of beauty all activate a brain region that lies within the vmPFC. "The experience of visual, musical, and moral beauty all correlate with activity in a specific part of the emotional brain, field A1 of the medial orbitofrontal cortex, which probably includes segments of Brodmann Areas (BA) 10, 12 and 32” (Zeki, 2014, p. 1). And “The experience of mathematical beauty correlates parametrically with activity in the same part of the emotional brain... as the experience of 
beauty derived from other sources” (p. 1). Summarizing, different kinds of beauty all activate the same region of the brain that is responsible for mental networks.

It should also be added that the findings of Zeki have only been partially replicated by later studies. For instance, a recent paper that examined beauty "confirmed the presence of a domain-general representation in aMPFC and AMPFC, and also revealed adjacent domain-specific regions in frontal pole and inferior/orbital frontal cortex, primarily for architecture and art” (Vessel, 2019, p. 19159). However, the authors did not find as strong a connection between beauty and the vmPFC as that reported by Zeki. Mental networks provide a possible explanation for this discrepancy. Zeki's study of mathematical beauty examined graduate students of mathematics who would have developed potent TMNs involving mathematics. Both Kawabata \& Zeki's (2004) study involving paintings and Ishizu \& Zeki's (2011) study of musical beauty presented subjects with a number of samples beforehand and then only used the ones that the subjects regarded as strongly beautiful or strongly ugly. Presumably, such pre-choosing would emphasize items that trigger existing mental networks within the subjects. In contrast, Vessel (2019) deliberately chose images which were unfamiliar to subjects, making it less likely that existing mental networks would be triggered.

However, a related article by the same group which examined how the brain response changes over time describes a "signal timecourse observed in anterior medial prefrontal cortex (aMPFC), a core node of the DMN, [default-mode network] where an initial suppression was followed by a rise several seconds later only for those artworks found most aesthetically appealing” (Belfi, 2019, p. 585). This suggests that art which is 'most aesthetically appealing' is triggering a mental network while normal art is not. Consistent with the idea of a mental network, the authors "suggest that increased activation of the DMN to high-rated artworks may instead reflect 'engagement' with the stimulus, by which we mean that a person is 'pulled in' to the stimulus, actively thinks about it, and chooses to continue mentally interacting with the stimulus” (p. 592). Notice how the viewer is being 'pulled in', indicating that a certain interpretation is emotionally imposing its structure upon the mind.

Zeki points out in another paper that education is required to appreciate mathematical beauty. "Those not versed in the language of mathematics cannot experience the beauty of a mathematical formulation. And yet, once the language of mathematics is mastered, the same formulae can be experienced as beautiful by mathematicians belonging to different races and cultures” (Zeki, 2018, p. 7). This suggests that there is a relationship between education and theoretical emotion. Some form of education is required to appreciate theoretical emotion.

The relationship between education and theoretical emotion can be seen in the 'aha' moments of education, in which the complexity of some topic suddenly fits together within the mind of the student. This is described in a paper on the education of mathematics. The paper begins, "The AHA! experience - the moment of illumination on the heels of lengthy, and seemingly fruitless, intentional effort - has long been the basis for lore in mathematics" (Liljedahl, 2005, p. 219). Notice that what is being described is not order but rather order-within-complexity, because an intense struggle with complexity is breaking through to the illumination of order. And the author describes the 'AHA! experience' as an emotion that is capable of changing existing MNs. "Beliefs about mathematics are often based on their 
own experiences with mathematics... A qualitatively different form of belief is with regards to a person's beliefs in their ability to do mathematics" (p. 221). "The AHA! produced more than simply a 'good' feeling. It contributed to a positive change in the beliefs” (p. 228). Notice how emotional experiences involving math have turned into mental networks and these mental networks have become part of self. An 'aha moment' is capable of altering these MNs.

This is a significant issue because "Approximately $17 \%$ of the US-American population suffers from high levels of math anxiety" (Luttenberger, 2018, p. 312). And math anxiety is related to mental networks. "Math anxiety describes an enduring, habitual type of anxiety and can be understood as a trait which represents a fairly stable characteristic of an individual and that influences how an individual feels in, perceives, and evaluates specific situations” (p.312). Notice how repeated emotional experiences involving math are leading to 'the stable characteristic of an individual', suggesting that MNs have formed which are being regarded as part of self. A neurological study found that "Effective connectivity between the amygdala and ventromedial prefrontal cortex regions that regulate negative emotions was elevated in children with math anxiety” (Young, 2012, p. 492). In other words, negative experiences involving math have formed MNs within the vmPFC. In addition, "Math anxiety was associated with reduced activity in posterior parietal and dorsolateral prefrontal cortex regions involved in mathematical reasoning” (p. 492). This indicates that the education required to appreciate the theoretical emotion of math is inadequate. Notice that the dorsolateral prefrontal region is adding factual content to mental networks. This is a significant function and the interaction between the dorsolateral prefrontal and mental networks will be discussed later.

It was suggested earlier that a mental network can form either as a result of a traumatic experience or after a period of time as a result of repetition. A traumatic experience is usually imposed upon the mind from the physical body. Theoretical emotion, in contrast, emerges when internal content comes together in some ordered manner. This suggests that theoretical emotion can lead to the formation of mental networks, but these mental networks will form as a result of repetition rather than traumatic experience. Stated succinctly, if some theory continues to be used, then it will eventually form a mental network. This type of mental network will be referred to as a TMN or theoretical mental network, in order to distinguish it from normal MNs that are based in experience. ${ }^{2}$ Like a normal MN, a TMN will emotionally impose its structure upon the mind when it is triggered. This will express itself as an emotional drive to use this theory to explain the current situation.

Habits were discussed earlier in the context of MNs. A habit should be viewed more accurately as a non-verbal form of TMN. When the same sequence of actions is repeated, then order can be brought to the complexity of these actions by representing them through some form of exemplary sequence. This sequence of actions turns into a habit when it becomes treated by the brain as a single 'chunk' and is associated with some mental network in the vmPFC. Habit formation also involves a shift from the caudate to the putamen in the basal ganglia (Patterson, 2018), but this paper is focusing upon mental networks and not how the content of mental networks is developed.

2 The term 'normal MN' will be used when referring to MNs based in emotional experience as opposed to TMNs. 'Mental network' will be used when emphasizing that both normal MNs and TMNs are being included. 
TMNs that come from verbal theories and TMNs based in nonverbal habits share several features: 1) they both emerge as a result of repetition; 2) they are both based in sequences; 3) the resulting TMN functions independently of normal emotions. This means that a TMN will motivate a theory to be used —or a habit to be performed — whether this leads to beneficial results or not.

Verbal theories and habits are combined in the exemplar, a concept introduced by Thomas Kuhn in the second edition of The Structure of Scientific Revolutions (Shan, 2020). On the one hand, science has developed extensive verbal and symbolic theories, often expressed through mathematics. On the other hand, Kuhn pointed out that a major facet of learning science is repeatedly performing the actions of solving science problems. Thus, learning science also involves building habits of solving science problems. Shan suggests that an exemplar has five main components: a vocabulary; a set of welldefined research problems; a set of practical guides; a set of models; and a set of patterns of reasoning. Vocabulary, models, and reasoning are aspects of verbal theories, while research problems and practical guides relate to sequences of actions being performed in a habitual manner.

\section{The Behavior of a TMN}

What distinguishes a TMN from theoretical emotion is the presence of an emotional drive. Learning some general theory can lead to a feeling of theoretical emotion, but this will not be accompanied by a drive to continue using this theory. However, if a theory continues to be used, then eventually a threshold will be crossed where the theory turns into a TMN. It will feel as if the theory has acquired a life of its own, similar to the way that a normal MN behaves like a living being. One will no longer choose to use the theory to explain situations but rather feel driven to use the theory. For instance, I remember the moment when the theory of mental symmetry turned into a TMN within my mind. I had been assisting my older brother (Lane Friesen) in his study of cognitive styles. This included analyzing my own thinking in order to gain a better understanding of my cognitive style. Suddenly, it felt as if the theory became alive, because my behavior was being followed a second or two later by an internal voice that analyzed this behavior. The 'aha moment' was discussed earlier. I suspect that an 'aha moment' can be accompanied by the formation of a TMN but that these are not necessarily connected. In my personal case, the TMN formed as I was driving my car and was not accompanied by any 'aha moment'.

It should be clarified that the reference to an 'emotional drive' is a slight simplification. More accurately, a TMN will generate a positive emotion if it is triggered and permitted to impose its 'explanation' upon some situation, while generating a negative emotion if it is triggered and prevented from imposing its structure upon some situation. These feelings will result in an emotional drive to continuing using the theory. In the same way that avoidance can be used to prevent MNs of addiction from being triggered, so it is possible to avoid the emotional drive generated by a TMN by not triggering the theory, which can usually be done by avoiding the terminology of that theory.

A similar emotional drive can be seen in a bureaucracy. Behavior within such a group is guided by a system of rules and procedures that bring order to the complexity of social interaction. Working within a bureaucracy will lead to the formation of TMNs within the minds of the members of a bureaucracy. 
This TMN will create an emotional drive to maintain the structure of the bureaucracy which is independent of any reason for applying the rules of the bureaucracy. Saying this more simply, a bureaucracy will develop a life of its own. The TMN of a bureaucracy will drive its members to apply the rules of the bureaucracy to a situation even when this application is unnecessary, illogical, meaningless, or overrides personal and social MNs. Saying this more simply, the TMN of a bureaucracy may feel inhuman because it functions independently of the MNs of normal human existence. Because theoretical emotion requires education, outsiders who interact with a bureaucracy will lack the training that is necessary to appreciate the theoretical emotion that lies behind the bureaucracy. They will merely notice that they are being forced to interact with some inhuman entity that is overriding their personal MNs.

A bureaucracy typically forms in the following manner: 1) some individual or group is motivated by personal MNs to behave in a manner that violates the MNs of morality and/or society; 2) a rule is formulated to ensure that this injustice does not happen again; 3) as the number of rules grow, they are eventually organized so that order can be brought to the complexity of regulations, leading to the establishment of a bureaucracy; 4) functioning within these rules forms a TMN, leading to the mindset of a bureaucrat; 5) this TMN will override other mental networks, causing the bureaucracy to function in an inhuman manner. Describing this progression another way, the development of rules can be viewed as a form of education. This is followed by the theoretical emotion of a bureaucracy and working within a bureaucracy leads to the formation of TMNs.

This progression has been noted by others (Lutzker, 1982): "Let us begin with that dreaded word, 'bureaucracy,' whose origins are rather innocent” (p. 120). 1) “Pre-modern administrative systems were characterized by inefficiency, nepotism and other kinds of favoritism, corruption, and coercion” (p. 123). 2) "It is against this background that one must measure modern systems of administration" (p. 123). 3) "Administration came increasingly to be entrusted to distant, more impersonal offices applying a central system of uniform decrees or laws" (p. 123). 4) "In time a new type of individual emerged onto the historical stage: the administrator, the civil servant whose task it was to carry out the will of the central authority" (p. 123). 5) "All of us have experienced the frustration of dealing with bureaucracy, and everyone has a favorite horror story” (p. 120).

The relationship between theoretical emotion, education, and a TMN can also be seen in ideology. "Ideologies are foundational social beliefs of a rather general and abstract nature. One of their cognitive functions is to provide (ideological) coherence to the beliefs of a group” (Van Dijk, 2006, p. 116). In other words, an ideology provides a theory that generates theoretical emotion. This theory turns into the TMN of an ideology as it continues to be used: "One does not become a pacifist, feminist, racist or socialist overnight, nor does one change one's basic ideological outlook in a few days. Many experiences and discourses are usually necessary to acquire or change ideologies” (p. 116). Acquiring this theory requires some form of education. An ideology "has more or less explicit, more or less formalized and institutionalized ways to teach ideologies to new members, e.g. special meetings, schooling, catechism, textbooks, propaganda leaflets, sermons, and so on” (p. 133). Finally, an ideology uses emotional means to impose content, consistent with a mental network. "Some ideologies 
may thus function to legitimate domination, but also to articulate resistance in relationships of power, as is the case for feminist or pacifist ideologies. Other ideologies function as the basis of the 'guidelines' of professional behaviour-for instance for journalists or scientists” (p. 117). It should be emphasized that we are not looking here at the legitimacy, accuracy, or appropriateness of an ideology. An ideology is not necessarily bad. Instead, we are merely noting that the formation and behavior of an ideology corresponds with the concept of a TMN.

In most cases, TMNs function as an overlay to normal MNs. The TMN then provides an emotional drive to universalize the emotions generated by the normal MN. For instance, cultural MNs will motivate a person to think and behave in a manner that is consistent with these MNs as well as causing a person to be emotionally attracted to others who behave in such a manner. The addition of a TMN will turn 'I want to protect my culture' into 'My culture defines how civilized people should behave'. Or for a more personal example, adding a TMN will turn 'That was a stupid thing to do' into 'You are a stupid person who always does stupid things'. Mathematics is an example of TMNs emerging within a context that is free of MMNs. Modern scientific thought also provides an example of reasonably pure TMNs, because science attempts to eliminate MNs of personal bias by remaining objective, and anyone who works within a scientific specialization will use a theory long enough for it to turn into a TMN.

For instance, a paper on ToM quoted earlier complained that "Our adult view is utterly contaminated by our own skill at mentalizing and there is no guarantee that infants think like we do" (Ruffman, 2014, p. 268). Looking at this cognitively, suppose that some individual studies ToM, develops theories about ToM, and then does research on the topic of ToM. This will cause the researcher's theories about ToM to turn into TMNs, and when this researcher studies ToM in the child, these TMNs within the mind of the researcher will attempt to impose their structure upon the mind of the researcher, causing the researcher to interpret childish ToM in terms of adult mentalizing.

Research into ToM has traditionally been divided into two major camps, with one side (simulation theory) saying that people simulate what is happened within other peoples' minds, and the other side (theory theory) saying that people use theories to predict what is happening within other people's minds (Apperly, 2008). We have seen how normal MNs naturally simulate the behavior of other people, and this type of ToM will tend to be prevalent when interacting with close friends or family members who are mentally represented by significant MNs. But a TMN can also be used to predict the behavior of some typical situation or some profession, such as 'how one behaves in a bank' or 'visiting the doctor'. In this case, one is coming up with general principles of behavior that bring order to some complexity of situations. These two options describe what is happening within the mind when using ToM. But added to this is the TMN in the mind of the researcher who is writing the academic paper on ToM, who developed a TMN as a result of thinking carefully about ToM. That TMN will naturally influence the researcher to interpret ToM as theory of mind, rather than simulation of mind.

This suggests that ToM involves both simulation and theory, and "Many authors now argue for a hybrid account in which both simulation and theory play a role” (Apperly, 2008, p. 268). However, this is not a matter of using MNs to respond quickly to a situation as opposed to using rational thought to respond slowly and carefully. That is a valid distinction which is summarized by dual process theory (Sloman, 
1996). Instead, both normal MNs and TMNs are functioning intuitively in a similar manner. A normal MN imposes the likes and dislikes of some person, group, or situation when it is triggered, while a TMN imposes some pattern of structured behavior when it is triggered. Often the response will be determined by some combination of normal MNs and TMNs, with the various MNs forming an emotional hierarchy that determines behavior. For instance, the schema of 'behaving in a bank' does not involve putting on a mask. But the schema of 'behaving in public' currently requires putting on a mask because of the Covid-19 pandemic. Visiting a bank now activates both of these MNs, causing the MN of 'behaving in a bank' to be modified by the MN of 'behaving in public'.

\section{TMNs, Paradigms, and Intuition}

The TMN provides an explanation for Thomas Kuhn's concept of paradigms and paradigm shifts (Kuhn, 1970). A paradigm can be defined as a theory that guides the thinking of some specialization: "Men whose research is based on shared paradigms are committed to the same rules and standards for scientific practice. That commitment and the apparent consensus it produces are prerequisites for normal science” (p. 11). Similar TMNs will form within the minds of those who use the same paradigm, making it possible for these researchers to "agree in their identification of a paradigm without agreeing on, or even attempting to produce, a full interpretation or rationalization of it. Lack of a standard interpretation or of an agreed reduction to rules will not prevent a paradigm from guiding research" (p. 44). In other words, working within a paradigm usually involves the application of rigorous thought, which Kuhn refers to as the 'puzzle solving' of normal science. But the paradigm itself functions at the less rigorous level of a general theory. Going further, logical problems are insufficient to cause a paradigm to be questioned. Scientists "may begin to lose faith and then to consider alternatives, [but] they do not renounce the paradigm that has led them into crisis. They do not, that is, treat anomalies as counter-instances” (p. 77). This is consistent with a TMN emotionally imposing the structure of the paradigm upon the mind. Paradigm shifts do occur, but the emotional influence of a TMN may be strong enough to prevent a scientist from ever making a paradigm shift. Kuhn quotes Max Planck as concluding pessimistically that "A new scientific truth does not triumph by convincing its opponents and making them see the light, but rather because its opponents eventually die, and a new generation grows up that is familiar with it” (p. 151).

We saw earlier when looking at impetus that the untrained mind tends to use MNs to interpret physical movement. Presumably, one of the purposes of education is to replace these naïve MNs with TMNs of rational understanding based in the paradigms of science. Using the language of process theory, this is normally interpreted as replacing Type 1 intuition with Type 2 analytic thinking. However, a paper that looked at errors of medical diagnosis concluded that "The theoretical position that errors in clinical reasoning arise primarily from Type 1 processing and are corrected by Type 2 processing is simplistic" (Norman, 2017, p. 29). That is because "General admonitions to slow down, reflect, or be careful and systematic likely have minimal effect beyond slowing the diagnostic process. By contrast, knowledge deficits are a significant contributor to diagnostic error" (p. 29). This suggests that merely thinking more carefully is often insufficient. Instead, education is required, which makes it possible to replace naïve MNs with TMNs that function intuitively but are more accurate. 
Looking at this more generally, intuition is related to vmPFC activity. A study of intuition found that "Activation shifted from the dorso-fronto-parietal network, which involves dorsolateral prefrontal cortex (DLPFC) for deliberate choice of novel CS [conditional stimuli], to ventro-medial frontal (VMPFC) and anterior cingulate cortex for intuitive choice of familiar CS” (Erdeniz, 2019, p. 174). And studies of expertise, such as Dreyfus' model of expertise, suggest that the expert is functioning primarily at an intuitive level (Dreyfus, 2004). This again suggests that expertise involves replacing naïve MNs with TMNs that have been acquired through education.

Similarly, a paper on schema quoted earlier describes vmPFC activity using language that sounds like expert intuition - which goes beyond experiences to general theories. "Reinstatement of even highly learned schemas strongly engages, and even requires, the vmPFC... Schemas... involve highly variable abstract representations of both the nodes and their interrelations, and under these conditions the vmPFC and its interactions with posterior cortices could be necessary even when no specific episodic information is retrieved" (Gilboa, 2017, p. 622).

However, a study of $\mathrm{PhD}$ level physicists suggests that they are functioning primarily at the level of using analytical thought to suppress naïve intuition rather than utilizing expert intuition. Using the language of process theory, they are replacing Type 1 intuition with Type 2 analytical thought. "High school and university students activate frontal brain areas associated with inhibitory control to overcome naive ideas in science, probably because they persist despite scientific training... Results suggest that naive ideas are likely to persist, even after completing a Ph.D. Advanced experts may still rely on high order executive functions like inhibitory control to overcome naive ideas when the context requires it” (Allaire-Duquette, 2021, p. 1). More specifically, this study did not find activity in the vmPFC, suggesting that mental networks are not guiding thought. This brings to mind Kuhn's observation that "The scientific enterprise as a whole does from time to time prove useful, open up new territory, display order, and test long-accepted belief. Nevertheless, the individual engaged on a normal research problem is almost never doing any one of these things... What then challenges him is the conviction that, if only he is skilful enough, he will succeed in solving a puzzle that no one before has solved or solved so well” (Kuhn, 1970, p. 38). In other words, even though science talks about paradigms and is ultimately based upon paradigms, most scientific thought functions at the technical level of manipulating and evaluating content rather than at the intuitive level of being guided by educated TMNs.

In contrast, "A large amount of data has been collected about intuition in expert nursing, and intuition plays an important role in the influential theory of nursing expertise” (Gobet, 2008, p. 129). The paper opens by saying that "Intuition is often proposed as one of the defining characteristics of expertise" (p. 129). However, "The role of intuition in nursing has been the topic of considerable debate, with some authors considering that this concept should be subjected to critical scrutiny at best and rejected at worst” (p. 130). In other words, nursing often functions at the level of expert intuition, possibly because nursing has to interact directly with MNs of human emotion. However, this use of expert intuition is regarded as academically suspect. The authors then present a model of expert intuition which can be summarized as emotionally imposed schemas, consistent with the concept of mental networks. These 
schemas probably include a combination of normal MNs and TMNs, just as ToM appears to involve a combination of normal MNs and TMNs.

Summarizing, the naïve mind thinks in terms of personal MNs, as illustrated by the concept of impetus. This naïve thinking is a holdover from the MNs of Piaget's precognitive stage. Education uses the rational thinking of executive function (largely from the dorsolateral frontal cortex) to override these naïve MNs. This type of careful rational thought tends to characterize technical fields such as physics. Working within some technical field will cause the paradigms of that field to turn into TMNs. These TMNs, combined with MMNs of experience, make it possible to function intuitively as an expert. This intuitive expertise will be more evident in fields such as medicine, while tending to be suppressed as insufficiently rigorous in fields such as physics. However, TMNs will become apparent even in more rigorous fields during paradigm shifts.

The distinction between naïve intuition and expert intuition can be seen in a study that examined vmPFC activity when acquiring information through articles in the New York Times. "People with less experience with the class of media we focused on tended to show better vmPFC tracking of consensus value and better tracking of media effects in the population... [but] lower connectivity of vmPFC with brain regions involved in controlled processing. This pattern suggests that vmPFC is most related to population behavior when it acts more independently from regions involved in top-down cognitive processing” (Doré, 2019, p. 3108). Stated cognitively, when MNs of societal opinion are used to evaluate information, then the vmPFC is operating largely by itself. However, when TMNs of expertise are used to evaluate information, then the vmPFC is functioning together with other brain regions that check information.

This suggests that learning can be divided into three stages. 1) The beginner: Naïve MNs guide the mind in an untrained and probably inaccurate manner. 2) The technician: Education programs executive function, especially within the dorsolateral frontal cortex, and this education is used to suppress naïve MNs. 3) The expert: Education leads to the formation of TMNs, which allow the expertise of the dorsolateral frontal cortex to be expressed in an intuitive manner.

The tendency for science to attempt to suppress naïve MNs rather than replace them with educated TMNs leads to strange results when science tries to study TMNs. That is because scientific thought is being emotionally driven to study the emotional drive behind scientific thought, guided by the premise that scientific thought eliminates emotional drive. For instance, Zeki's neurological study of beauty was quoted earlier when introducing the concept of a TMN. A later paper by Zeki opens by rejecting historical concepts of beauty and replacing them with a sweeping statement. (The sweeping statement will be discussed in the next section.) "There have been many definitions of beauty, but none of them is wholly or even partially satisfactory. My favourite is the one given by Edmund Burke, partly because it says nothing and partly because it says everything.” (Zeki, 2019, p. 107). Zeki then proposes a circular definition of beauty. "Beauty is an experience that correlates quantitatively with neural activity in a specific part of the emotional brain, namely, in the field A1mOFC; the more intense the declared experience of beauty, the more intense the neural activity there” (p.109). Thus, the neurological finding that beauty activates a certain part of the brain is now being used to define beauty. 
Something similar happened to Thomas Kuhn. On the one hand, “Kuhn's introduction of the concept of paradigm... in SSR [The Structure of Scientific Revolution] is his most novel and significant contribution to the philosophy of science" (Shan, 2020, p. 383). On the other hand, "After 1970 Kuhn seldom used the concept of paradigm or exemplar to analyse the history of science" (p.386). Shan describes this about-face as 'extraordinary'. Kuhn is regarded as "one of the most influential and important philosophers of science in the second half of the twentieth century" (p. 381) and yet he turned his back upon the primary reason for his fame. My goal in mentioning these examples is not to minimize the research of these individuals but rather to illustrate what happens when the current scientific paradigm of objective thought meets itself.

\section{The TMN and a Concept of God}

The same suppression of naïve MNs that occurs in physics can also be seen in the relationship between theology and religion. One of the core findings of the cognitive science of religion (CSR) is that subjects "use anthropomorphic concepts of God in understanding stories even though they may profess a theological position that rejects anthropomorphic constraints on God and God's activities. It appears that people have at least two parallel God concepts that are used in different contexts, and these concepts may be fundamentally incompatible” (Barrett, 1996, p. 240). An 'anthropomorphic concept of God' refers to a normal MN (possibly amplified by a TMN) which represents the imaginary person of God as some sort of human-like Superman. Theology uses education to attempt to suppress these naïve MNs of god-as-superman, but they still function at an intuitive, implicit level. This is like the trained physicist who also uses training to suppress naïve MNs that still function at an intuitive, implicit level.

Behind these findings lies a deeper question. Why do science and theology exist? CSR uses the concept of the 'hyperactive agency detector device' (which corresponds to an $\mathrm{MN}$ ) to explain the natural tendency to anthropomorphize a concept of God (Barrett, 2007). MNs can explain how a concept of God becomes misinterpreted, but what is causing a concept of God to emerge in the first place? Similarly, we saw earlier that "High school and university students activate frontal brain areas associated with inhibitory control to overcome naive ideas in science” (Allaire-Duquette, 2021, p. 1), but what is causing science to emerge in the first place?

Kuhn says that a paradigm is required for normal science. Repeating an earlier quote, "Men whose research is based on shared paradigms are committed to the same rules and standards for scientific practice. That commitment and the apparent consensus it produces are prerequisites for normal science” (Kuhn, 1970, p. 11). And a paradigm can be explained using the concept of a TMN. The TMN of a paradigm may function under the surface during normal science with its technical puzzle-solving, but this TMN is responsible for the existence of normal science, and it becomes evident when the paradigm is challenged or threatened. Similarly, I suggest that the TMN is responsible for the existence of theology. This TMN may be functioning incompletely in many who profess to believe in theology, but it provided the emotional motivation for inventing theology, and it provides emotional motivation for maintaining theology. 
These two methods of forming a concept of God can be seen in two quotes by Paul Dirac, the famous physicist. Dirac stated at a conference in 1927 that "If we are honest-and scientists have to be-we must admit that religion is a jumble of false assertions, with no basis in reality. The very idea of God is a product of the human imagination. It is quite understandable why primitive people, who were so much more exposed to the overpowering forces of nature than we are today, should have personified these forces in fear and trembling. But nowadays, when we understand so many natural processes, we have no need for such solutions" (Heisenberg, 1971, pp. 85-86). Notice how Dirac is reacting to a religious mindset that is functioning at Piaget's precognitive level. Traumatic experiences with nature are forming MNs which are being interpreted as living beings. Theoretical emotion is then generalizing from these traumatic experiences, causing TMNs to be intuitively overlaid upon these MNs, resulting in traumatic experiences being interpreted as gods. Dirac points out that the honesty of science forces it to challenge these mental networks, which was seen earlier in trained physics students mentally suppressing naïve MNs.

Looking at the thinking of the scientist more carefully, it was suggested earlier that mental networks form an emotional hierarchy, with more potent mental networks imposing their structure upon lesser mental networks. A student of theology or physics has acquired TMNs as a result of education. These TMNs will gain sufficient emotional potency within the minds of some experts to override naïve MNs, providing a motivation for developing further theoretical understanding, but for the average student, naïve MNs remain emotionally more important than TMNs of education, meaning that significant mental effort still has to be used to suppress these naïve MNs when attempting to be theologically or scientifically correct.

The presence of a motivating TMN in an expert can be seen in the second quote by Dirac. Later in life, he stated that "It seems to be one of the fundamental features of nature that fundamental physical laws are described in terms of a mathematical theory of great beauty and power, needing quite a high standard of mathematics for one to understand it. You may wonder: Why is nature constructed along these lines? One can only answer that our present knowledge seems to show that nature is so constructed. We simply have to accept it. One could perhaps describe the situation by saying that God is a mathematician of a very high order, and He used very advanced mathematics in constructing the universe" (Dirac, 1963, p. 52). Dirac starts with the concept of mathematical beauty discussed earlier. He then observes that this mathematical beauty appears to be a property of nature that exists independently of the mind. Studying this mathematical beauty caused TMNs to form within his mind which he interprets as a concept of God. Notice that the emotional hierarchy has shifted. In the first quote, Dirac complained about concepts of gods formed by TMNs that extrapolate from existing MNs. In the second quote, Dirac is describing a concept of God that is based in TMNs of mathematics which function independently of existing MNs.

This leads to the following definition: A concept of God emerges when a sufficiently general theory turns into a TMN and is interpreted personally. This definition does not say how this general theory is formulated, and different ways of formulating a general theory will lead to different concepts of God. If a theory is insufficiently general, then what will emerge instead is a concept of many gods based in 
TMNs that extrapolate from existing MNs of culture, race, or profession. This can be seen in the Greco-Roman gods who were essentially amplified versions of normal humans. One does not find many people today advocating for the existence of such gods. Instead, the discussion is over the existence of God. This suggests that the theories of science have become sufficiently general and sufficiently well-known to set the context for thinking about God. Thus, the TMNs of scientific understanding may not be sufficiently developed within the mind of the average person for that individual to conclude that 'God is a mathematician', but they have changed the topic of discussion from gods to God.

Notice also that this definition focuses upon a mental concept of God. This definition makes it possible to study and compare different concepts of God from a cognitive and psychological perspective without having to determine whether or not such a God actually exists. And even if God does not exist, the TMN of a concept of God has sufficient emotional power to guide the course of societies and civilizations, making it societally meaningful to analyze concepts of God. Going further, it is possible to assign moral value to different concepts of God: a concept of God that encourages cognitive development and integration can be regarded as morally superior to a concept of God that discourages cognitive development and leads to mental fragmentation. Finally, this definition is internally consistent because one is viewing TMNs from a theoretical perspective, rather than examining MNs of religious experience and then extrapolating from that to concepts of God. Such internal consistency is necessary because we saw earlier when looking at ToM that the mental networks of the researcher have a natural tendency to impose themselves upon the interpretation of the data.

The presence of a TMN can also be seen by examining the standard theological concept of God. Barrett, a researcher in the cognitive science of religion, provides a good summary: "In contrast to the ancient Greeks, contemporary Western theologies suggest the existence of a gaping ontological chasm between God and humans... God is not simply more of what we are. There is an essential discontinuity. One way to appreciate this 'essential discontinuity' is to consider the descriptions of God offered by the three monotheisms that have most influenced Western thought about God. Catholic and Protestant teachings describe God as being: infinite, limitless, all-perfect, all-powerful, unchanging, nonmaterial, all-knowing, and perfectly simple. Similarly, Judaism speaks of God as omnipotent, omniscient, omnipresent, and eternal. God is neither bound nor limited by space or physical laws. Within Islamic theology the same essential themes are repeated: human existence is entirely different than God's” (Barrett, 1996, pp. 219 - 220).

Stated cognitively, Greek gods were 'more of what we are'-MNs of culture and nature amplified by TMNs. Monotheism, in contrast, is the ultimate example of a sufficiently general theory being interpreted personally, because it states that behind the complexity of the entire universe lies a universal being known as God who brings order to this complexity. Looking at this in more detail, it was mentioned earlier that an MN functions like a living being and the mind uses MNs to represent living beings. Thus, it makes sense that a TMN would also be interpreted as a living being. But a TMN comes from a theory that brings order to the complexity of situations, and theories have to be taught to be appreciated. This combination leads to the concept of a universal being who transcends specific 
experiences and situations together with the concept that people must be educated to learn about this universal being. This will be expressed as a monotheistic religion accompanied by missionary activity that emphasizes education, supported by books that teach about God.

We have seen that a theory with its theoretical emotion can be distinguished from any careful thinking that is used to develop or test this theory. For instance, a paradigm makes normal science possible, but normal science focuses upon solving technical puzzles within this paradigm, and normal science will ultimately reject a paradigm if sufficient anomalies emerge that cannot be solved by technical puzzle solving (Kuhn, 1970, p. 83). Because the mental circuit that generates theories is distinct from the mental circuit that checks theories, it is possible to form a general theory by making a sweeping statement and then disregarding any information that might contradict or limit this sweeping statement. Stated simply, one is forming a theory but then neglecting to check it.

This combination can be seen in Barrett's summary of monotheism quoted a few paragraphs earlier. Most of the terms that Barret uses are sweeping statements that transcend specific factual information: infinite, limitless, all-perfect, all-powerful, unchanging, all-knowing, perfectly simple, omnipotent, omniscient, omnipresent, and eternal. The statement of 'nonmaterial' suggests that theoretical emotion is being generated internally, independent of physical evidence. And the separation between a sweeping statement and specific facts can be seen in the 'gaping ontological chasm between God and humans' that separates a concept of God from the facts of human existence as well as the statement that 'human existence is entirely different than God's'.

There is some evidence linking the vmPFC to a concept of God. A study of war veterans with brain damage found that "damage to the right vmPFC resulted in a stronger personal relationship with God" (Cohen-Zimerman, 2020, p. 575). This brings up the matter of brain laterality. It is possible that normal emotion is related to the right hemisphere and theoretical emotion to the left hemisphere. This makes intuitive sense because most speech involves the left hemisphere and theories are typically constructed using words. However, distinguishing between these two emotions is not straightforward because most situations can be approached from either an experiential or a theoretical perspective. For instance, self can be defined either as a collection of normal MNs composed of experiences with emotional labels, or as a set of TMNs that summarize personal skills and knowledge. An experiential perspective might think about Fred the next-door neighbor who works as a butcher, while a theoretical perspective would think about the profession of butcher of which Fred is a specific example. Returning to the vmPFC, few papers attempt to differentiate left from right vmPFC. One study of teenage boys found that "the right vmPFC was a significant predictor of impulse control ratings' (Boes, 2009, p. 1), which could be interpreted as normal MNs imposing their structure upon teenage behavior. We began our discussion of TMNs by looking at Zeki's study of beauty and the vmPFC. His data indicates that most of the brain activity was lateralized to the left vmPFC. If this lateralization is valid, then damage to the right vmPFC would impair the function of normal MNs, forcing a person to find emotional comfort by interacting socially with the TMN of a concept of God. Even if this is not the case, the paper by CohenZimerman indicates that it is neurologically appropriate to mention a concept of God within the context of discussing vmPFC function. 


\section{Mysticism and Awe}

As far as I can tell, all current religions form concepts of God by making sweeping statements that extrapolate beyond factual information. However, while all religions make a 'leap of faith', the size and nature of this leap varies considerably between one religion and another. At the most basic level, a distinction can be made between mysticism and theology. We will look first at mysticism before turning to theology. Mysticism can be explained cognitively as a combination of overgeneralization and identification. We saw earlier that "Identification involves a loss of one's sense of self" and is associated with the vmPFC (Broom, 2021, p. 542). Overgeneralization makes a sweeping statement that transcends specific factual information. Looking at this more carefully, if theoretical emotion is based upon order-within-complexity, then the greatest theoretical emotion can be generated by coming up with a universal theory that brings order to all of the complexity of all existence. The easiest way to generate such theoretical emotion is by making a sweeping statement about universality and then asserting that this statement transcends all factual information. This will form a powerful image of God if the concept of cosmic unity is interpreted as some sort of living being and if self identifies with this mental concept. Continuing to think about identification with cosmic unity will eventually lead to the formation of a powerful TMN, turning this into the concept of a mystical God that has sufficient emotional power to shape thought and behavior.

It should be emphasized that merely stating 'Make me one with everything' is insufficient. First, theoretical emotion requires education. Thus, one has to learn and think about the relationship between identity and the cosmos. Second, theoretical emotion comes from order-within-complexity. Thus, one must be aware of the complexity of personal existence and view cosmic unity as a source of order for this complexity. Third, a TMN takes time to emerge. Therefore, one must struggle with the relationship between identity and cosmic unity until 'breaking through' to the formation of a TMN.

Most of these factors can be seen in the three-factor model of mysticism (Streib, 2020). This model associates mysticism with eight statements, all beginning with "I have had an experience/I have experienced" (p. 6). This focus upon personal experience satisfies the requirement for personal interpretation. Looking at the eight statements, an "experience which was both timeless and spaceless" is not limited by factual information about reality (p. 6). An "experience in which something greater than myself seemed to absorb me” describes a combination of identification and generalization (p. 6). “An experience which cannot be expressed in words" results from an overgeneralization that goes beyond the factual categories of speech (p. 6). "An experience in which all things seemed to be conscious" indicates the presence of mental networks (p. 6). "An experience in which I realized the oneness of myself with all things" explicitly describes the combination of identification and overgeneralization (p. 6). An experience of "profound joy" describes an internally generated emotion that is not rooted in any specific situation. "An experience which I knew to be sacred” interprets this as a religious experience (p. 6). And "An experience in which a new view of reality was revealed to me” suggests that a theory of reality is behaving as a TMN (p. 6).

We saw earlier that scientific thought uses executive function to suppress naïve MNs. In a similar manner, evidence suggests that using executive function suppresses mystical thought. "Our findings 
support previous speculation linking executive brain functions to mystical experiences, and reveal that executive functioning (dlPFC) causally contributes to the downregulation of mystical experiences” (Cristofori, 2016, p. 212). If rational analysis suppresses mysticism, then this means that mysticism, by its very nature, must reject rational explanations_-including the explanation being given in this paper.

A concept of God that is based in mysticism cannot interact with the experiences of human existence because it is formulated by avoiding facts of human existence. Theology, in contrast, makes some statements about human existence and then takes a mental leap from this content to the sweeping statement that God transcends this content. A similar mental leap from some factual evidence to sweeping statement can also be seen in many ideologies. The precise nature of the factual content will vary from one system of theology or ideology to another and will generally be some combination of religious dogma, psychological principles, social constructs, and scientific laws. Thus, an ideology or theology is not automatically wrong, but it is characterized by a theoretical leap from partial information to sweeping statement which may or may not be valid.

Continuing to think about some system of theology or ideology will cause a TMN to form. This TMN may be based in the content of the ideology. But it is also possible for a TMN to form based upon the leap from rational content to sweeping statement. This focus upon a 'leap of faith' can emerge either out of mysticism or out of theology. Mysticism turns into a leap of faith when it has to coexist with science. Instead of approaching God by denying all facts of physical existence, God will be viewed as transcending facts about human existence. This can be seen, for instance, in the philosophy of Martin Buber (2012). Theology turns into a leap of faith when the content of theology is regarded as secondary and the jump from content to sweeping statement is regarded as primary. This can be seen, for instance, in the existential leap of Kierkegaard (McKinnon, 1993). Religious faith will then be interpreted as choosing to believe in God despite rational facts. Notice that one is dealing again with an emotional hierarchy of mental networks, because the TMN of 'the leap' is being regarded as more general—and thus possessing greater emotions of order-within-complexity - than other mental networks.

A leap into generality can also be seen in the experience of awe. Awe "has two core defining features. First, awe is induced by stimuli that are vast, including something immense in size, number, scope, complexity, ability, or social bearing... Second, awe stimulates a need for accommodation; in other words, when experiencing awe, individuals are forced to adjust their mental schema and alter their understanding of the world” (Guan, 2019, p. 1). The first feature describes the realization that self is merely one aspect of some much larger structure of order-within-complexity. This happens when TMNs of generality include MNs of self within their structure. But in the case of awe there is no TMN. Instead, the second factor indicates that existing TMNs are being revealed as inadequate. Thus, the mind is being forced by external stimuli to make a leap of accommodation into generality. One study found that "while watching awe videos, participants were deeply immersed in the videos and that levels of self-reflective thought were as much reduced during the awe videos” (Van Elk, 2019, p. 3561). Notice that the emphasis is not upon acquiring a TMN but rather upon realizing that existing mental networks are inadequate. For instance, one common example of awe is an astronaut seeing the entire 
globe from space for the first time; it becomes visually apparent that all MNs of self and social interaction are encompassed within a few specks of this single visual image.

Both Guan and Van Elk found that an experience of awe did not activate the medial prefrontal cortex, consistent with the idea that mental networks are not being activated. In contrast, a study that looked at spiritual feelings in devout Mormons found that "a recognizable feeling central to their devotional practice was reproducibly associated with activation in nucleus accumbens, ventromedial prefrontal cortex, and frontal attentional regions” (Ferguson, 2018, p. 104). This suggests that normal religious feelings involve the vmPFC, as opposed to feelings of awe, which are being imposed by the environment.

Awe can be separated into positive awe and negative awe. "Although positive and negative awe both arise in response to vast and complex stimuli... positive awe is characterized by greater feelings of calm states, and tonic positive affect, higher appraisals of personal control over the situation. While negative awe experiences are associated with greater feelings of fear and powerlessness, lower in self-control and certainty, and higher in situational control” (Guan, 2019, p. 2). This suggests that awe involves an emotion that is independent of the normal emotions of self. Saying this more carefully, both positive and negative awe share the common trait of order-within-complexity, which indicates that awe is related to theoretical emotion and not to normal experiential emotion. But positive awe supports MNs of self while negative awe threatens self, which indicates that the theoretical emotion of awe may be accompanied by either positive or negative experiential emotion.

Positive awe can be seen in the contemporary Christian practice of 'praise and worship' which occupies the first half of the typical charismatic Christian 'worship service'. One book on the subject introduces itself by saying that "Few things influence Christians' understanding of the faith more than the songs they sing in worship. The explosion of praise and worship music in the last fifteen years has profoundly affected our experience of God” (Woods, 2007). The term 'praise and worship' means in practice that praise songs will be sung first and then followed by worship songs. (Barrier, 2016). The goal of praise is to create a feeling of awe by encouraging audience immersion within powerful songs played by a powerful band, preferably within a large, full auditorium. This is then followed by worship, in which quieter songs are played that emphasize God's acceptance of self and love for self. This turns the awe into positive awe, leading to 'greater feelings of calm states, and tonic positive affect'.

Turning to another point, I suggest that a distinction needs to be made between an experience of awe or mysticism and the aftermath of such an experience. We have seen that an experience of awe appears to transcend existing mental networks. Similarly, "There is more or less a consensus that any experience appropriately qualified as mystical diverges in fundamental ways from ordinary conscious awareness and leaves a strong impression of having encountered a reality radically different from the sensorybased world of everyday experience. Rare and fleeting though they usually are, such experiences often stand out as joyous, defining moments” (Wulff, 2014, p. 369). Summarizing, one 'encounters a reality radically different from everyday experience' in a 'rare and fleeting' moment which 'leaves a strong impression'. Thus, the mystical experience itself is a rare event that appears to transcend existing 
mental networks, but having a mystical experience will lead to the formation of a TMN. ${ }^{3}$ Going further, thinking back upon transcendental experiences of awe and/or mysticism will form a TMN, and this TMN will motivate activity. For instance, the mystic will emphasize that the mystical experience transcends rational language, but many tomes have been written about mysticism which use rational language to attempt to describe that which transcends language (Yaden, 2016). It is not logical to write about something that transcends words, but the formation of a TMN provides a cognitive explanation for this illogical behavior. A similar distinction can be seen in the paper on Kierkegaard referenced earlier (McKinnon, 1993). The author points out that the phrase 'leap of faith' cannot be found in any of Kierkegaard's writings. However, this phrase is now used to summarize the teaching of Kierkegaard. Thus, an experience that transcended rational language for Kierkegaard has been reduced to the level of a TMN based in rational language by those who study the writings of Kierkegaard.

\section{Theology}

It was mentioned earlier that a concept of God which is formed by transcending the facts of human existence is incapable, by definition, of interacting meaningfully with human existence. A theological God, in contrast, contains sufficient content from human existence to interact with human existence. A theological concept of God still involves a 'leap of faith', but the focus will be upon extrapolating from human existence rather than transcending human existence.

It was also mentioned earlier that mental networks make it possible to have social interaction with an imaginary person. How a person interacts socially with a concept of God can be explored by looking at the traits mentioned at the beginning of this paper and replacing normal MNs with TMNs. Remember that a TMN is based in feelings of generality that come from order-within-complexity, and a concept of God forms when a sufficiently general theory applies to self. An MN activates relevant and suppresses irrelevant schematic information (Gilboa, 2017). Similarly, a person will 'see God' in the way that relevant events appear to come together in some meaningful manner, and generalizing and personalizing the concept of schema will lead to the feeling that 'God has a plan for my life'. MNs play a role in associating events with emotional responses (Lin, 2016). This leads to the feeling that 'God guides me in responding to the emotional experiences of life'. An MN causes a person to jump to conclusions (Brod, 2019). Similarly, a person will intuitively feel that 'God is guiding my thoughts and actions'. An MN generates an intuitive feeling of rightness (Hebscher, 2016). Similarly, 'following God' will generate an intuitive feeling of rightness. MNs cause a person to be attracted to others with similar MNs, leading to the concept of cultures (Kühn, 2010). Similarly, a person will be attracted to others with similar concepts of God, leading to the concept of faiths. An MN imposes its structure upon personal behavior (Bowren, 2018). Similarly, 'God expects you to have faith and act upon your beliefs'.

Looking at a more general characteristic, an MN will impose its structure upon the mind when it is triggered (Mi, 2021). Similarly, religious feelings will depend upon the context and a concept of God

3 It is possible that the formation of a new TMN based in a combination of overgeneralization and identification is the mystical experience. Such a TMN can only be formed by regarding existing mental networks as inadequate. 
will only affect thought and behavior when it is triggered. This leads naturally to a concept of holy people, holy places, and holy rituals, because both theological education and interaction with a concept of God will naturally be done within a context that triggers a concept of God, and religious adherents will find it difficult to maintain theological beliefs when functioning within a 'secular world' that is outside of this religious context. Notice that this limitation is not inherent with a concept of God but rather stems from the 'leap of faith'. A theological concept of God is being formed by extrapolating from a limited set of facts. Such a concept of God will only affect normal existence in areas where it connects with facts of normal existence. In other contexts, the sweeping statement will cause a cognitive disconnect to emerge between verbally claiming that God is omnipresent, omniscient, and omnipotent and acting as if God is not present, not aware, and powerless.

An MN can lead to addictive behavior (Miller, 2017). Similarly, the TMN of a concept of God has historically motivated many individuals to become monks or follow God in 'full-time service'. This also stems from the 'leap of faith'. That is because the discrepancy between making sweeping statements about God and having a concept of God that only applies to some of personal existence can be addressed by attempting to live only within the aspects of personal existence to which a concept of God applies. This becomes difficult when following a concept of God that is heavily biased towards mysticism, because following God fully will be interpreted as denying physical existence as fully as possible. This type of ascetism can be seen in the Desert Fathers who founded Christian monasticism. For instance, Saint Anthony remained within the walls of an abandoned Roman Fort in the Egyptian desert for twenty years, surviving on food thrown to him over the wall. A less strenuous form of 'fulltime service' becomes possible when various places, people, and rituals become mentally associated with a concept of God, because following God fully can then be interpreted as being one of the holy people who lives within these holy places and performs holy rituals.

Finally, it was mentioned earlier that an MN can motivate a person to 'fight and die' for their culture or beliefs (Pretus, 2019). A mental network will generate strong negative feelings if it continues to receive input that is inconsistent with its structure, because this repeated inconsistency will threaten the existence of the mental network. The emotional pain of threatening a core mental network can override other emotions causing a person to be willing to 'fight and die for their culture or beliefs'. When MNs of culture are being threatened, then the source of this threat is usually apparent. Typically, some ethnic group is being faced with genocide and is struggling for existence.

Threatening the existence of a TMN can also motivate a person to be willing to 'fight and die'. The cause for this threat will be apparent if the holy places, people, and practices of some religion are being attacked. However, suppose that there is a massive shift in the system of education of some society. This may happen over time as one generation replaces another, or it may happen as a result of being colonized or conquered by another society. The shift in education will mean that the new group will acquire a different set of TMNs than the old group, and the new dominant group will be motivated by its TMNs to behave in a manner that is repeatedly inconsistent with the TMNs of the original society. This repeated inconsistency will create an existential pain in members of the original society that is sufficient to motivate some of them to 'fight and die for their cultural beliefs'. But because the new 
group has not received the education of the original group, the new group will find the radical response of the original group incomprehensible. For instance, this explains the response of religious fundamentalists to the rise of postmodern society or the response of many aboriginal groups to Western society. The group whose TMNs are being threatened may feel willing to 'fight and die', while the outsider who received a different education will view this response as incomprehensible because no visible institutions are being threatened. Instead, what is being threatened is an internally generated TMN based in a certain form of education.

It should be emphasized that explaining interaction with God from a cognitive perspective does not automatically render such interaction invalid or prove that God does not exist. It could also be argued that a real God created the human mind to function in such a fashion. This is different than mysticism where any cognitive explanation will end up explaining it away. However, if a leap of faith is regarded as primary, then a cognitive explanation of interaction with God will explain away religious faith because a rational explanation removes the need for a leap of faith.

It should also be added that it is not possible to go beyond a theological concept of God with its inherent leap of faith when factual understanding is limited, because one has to extrapolate from limited data. However, a theological concept of God is inherently unstable, because it juxtaposes rational thought based upon factual information with sweeping statements that go beyond factual information. This means that the acquisition of new factual information will lead to one of two primary responses. Either the concept of God will be updated to include this factual information, or the information will be mentally bypassed by focusing upon the leap of faith. An example of attempting to update one's concept of God can be seen in the scientific writings of the $18^{\text {th }}$ century theologian Jonathan Edwards (Riforgiato, 1972), while focusing upon the leap of faith can be seen in the fundamentalist tendency to define faith as 'believing despite the facts'.

The leap of faith is not just a characteristic of right-wing religion. I suggest that it can also be seen in the left-wing doctrine of universal tolerance. For instance, one author distinguishes between toleration and tolerance, “defining 'toleration' as a set of social or political practices and 'tolerance' as a set of attitudes” (Murphy, 1997, p. 593). Toleration involves careful thought and factual analysis: “The calculus that goes into a toleration decision involves weighing the disapproval of one thing (the thing tolerated) against the disapproval of other things, including the unpleasant actions necessary to prohibit that thing and competing values violated in the process of prohibition” (p. 596). In contrast, the author defines "tolerance as a willingness to admit the possible validity of seemingly contradictory viewpoints, a hesitancy to pass value or 'truth' judgments on individual or group beliefs” (p. 600). Thus, tolerance avoids examining factual details. Tolerance replaces factual analysis with sweeping statements that transcend factual analysis: “Committing ourselves to a 'tolerant society' may offer a vision of inclusion and harmony. Recall, however, the vast range of definitions scholars offer for the concept. The amorphous quality of the term 'tolerance' often leaves observers wondering what a tolerant society would look like” (p. 618). Summarizing, in the same way that a theological concept of God juxtaposes factual content with a leap of faith, so modern social justice juxtaposes the factual analysis of toleration with the 'leap of faith' of tolerance. 
We saw when looking at mysticism that a sweeping statement is threatened by all rational analysis, even rational analysis that is being guided by the sweeping statement. That is because rational analysis uses a form of thinking that is, by its very nature, opposed to sweeping statements. One paper describes this kind of response in the current mindset of wokeness, which the author defines as "being aware of racial, gender, and economic injustice” (Jacobs, 2017, p. 35). This definition suggests that wokeness means being emotionally aware of contradictions to the concept of universal tolerance. The author describes the relationship between sweeping statement and rational analysis by making a distinction between the 'mythical core' and the 'technological core' of society, explaining that "One cannot analytically pick apart a complex, integrated mythical framework and say, 'I choose this but not that' without tearing holes in the web and leaving it dangling and useless... The attempt to translate mythical intuitions into 'rationalized structures' will therefore be perceived as a kind of violation” (p. 36). In other words, using rational analysis upon a myth automatically violates the myth. The author observes that wokeness is a form of myth that avoids rational analysis. "To describe wokeness in relation to the mythical core helps us understand why it is so fruitless to reply to the passionate student protesters with the commonplace distinction between means and ends” (p. 35). And when rational analysis is applied to the myth of openness, then this will lead to emotional rejection, even if this rational analysis supports the general premise of wokeness. Someone who uses rational analysis "may think of himself as a friendly, sympathetic, even supportive questioner, one who completely accepts the ends for which his interlocutors stand but has some questions about the best means for achieving them. He is therefore surprised when his questions meet with outrage and resentment” (p.35). Summarizing, wokeness is a form of universal tolerance that is based in a leap of faith, suggesting that the leap of faith is a cognitive mechanism which transcends political divisions between right and left.

\section{Fundamentalism}

The previous section looked at the situation of a new system of education being taught to the next generation or introduced by some colonizing or conquering group. Fundamentalism is an attempt to maintain the old system of education. In the words of one author, "What appears to be a basic criterion persisting across all types of (at least) monotheistic fundamentalism [is] a particular stance taken by fundamentalists toward the sacred text of their tradition” (Williamson, 2010, p. 722). In other words, there is a text or book. It is regarded as sacred or holy. It is the sacred text of some group. The group practices monotheism. The book helps maintain the traditions of the group.

Books have historically played a key role in education and education is required to appreciate theoretical emotion. Therefore, if a book describes a theory, then studying this book will lead eventually to the formation of a TMN. But a book is also a physical example of a theory. That is because it is composed of a complexity of words that have been ordered into sentences, paragraphs, and chapters, and then packaged into the physical object of a book. Education typically begins with a teacher handing a book to the student and saying, 'Here is your textbook. It contains everything you need to know.' Education hopefully ends with the student saying, 'I have studied the book and I now understand what it says.' Thus, the physical order-within-complexity of a book becomes transformed into the mental order-within-complexity of an internal understanding. A student will initially associate a 
book with MNs that represent respected authorities of society-augmented by the feeling of theoretical emotion generated by the book itself. The student will (hopefully) eventually associate the book with the TMN of an understanding.

A holy book adds a leap of faith to the textbook. A concept of God emerges when a sufficiently general theory is interpreted personally. A holy book takes a leap of faith from the physical order-withincomplexity of a book to universal statements about God. The teacher says to the student, 'This book tells you everything you need to know about God'. If this book describes a sufficiently general theory that applies to self, then this statement is a leap of faith that extends from partial information. The student will study the book, develop the TMN of a concept of God, and conclude that the book is an accurate description of universal truth. But it is also possible to focus upon the leap of faith that is being made from the specific object of the book to universal statements about God. The student who studies the book will still develop the TMN of a concept of God, but will conclude that the book is a holy book which is the sole source of absolute truth. A holy book is implicitly related to monotheism because a book implies education and packaging all significant knowledge within the object of a single book implicitly conveys the idea of a single universal theory.

Looking at this in more detail, Williamson uses six characteristics to describe how fundamentalists view their sacred text: 1) "Divine... The text is not a product of the human mind" (Williamson, 2010, p. 723). This describes the leap of faith in terms of mental networks. The authorship of a book is normally connected with a MN representing some human author. In this case, the TMN that represents God is being viewed as the author. 2) "Inerrant... [it] contains no inconstancies, errors, or contradictions whatsoever" (p. 723). The premise is that one can leap directly from the book itself to the TMN of a universal theory without requiring any error-checking from executive function. 3) "Self-interpretiveThe sacred text is sufficient in and of itself for understanding the divine intent and meaning of the author" (p. 723). This directly describes the leap from the specific words of a holy book to universal theory. 4) "Privileged-The sacred text is given a privileged status above all other texts" (p. 723). This means that the TMN of the holy book transcends the TMNs of all other theories and paradigms. 5) "Authoritative-The sacred text is considered to be authoritative” (p. 724). Because the TMN of the sacred text transcends other mental networks, it will impose its content upon other mental networks. 6) "Unchanging-The sacred text is immutable and timeless; thus it never changes" (p. 724). Thus, the leap from specific book to the TMN of a universal theory will not be affected by any changes to other mental networks.

However, similar statements could be made about a physics textbook. If one views God as the ultimate mathematician — as the older Dirac did, then a physics textbook could be viewed as being written ultimately by God. A physics textbook is reasonably inerrant, and studying merely the textbook will give a fairly complete understanding of natural processes. A physics textbook is legitimately given a privileged status above other books and it should be viewed as authoritative. And one of the basic premises of physics is that the laws of physics are independent of time and location. Thus, as far as a student is concerned, there is not much difference between a textbook and a holy book. In the words of Thomas Kuhn, "Science students accept theories on the authority of teacher and text, not because of 
evidence. What alternatives have they, or what competence?” (Kuhn, 1970, p. 80). The primary distinction arises in how the book is taught and learned. If the physical object of the book is regarded as the source of truth, then this will lead to the concept of a holy book. If the book describes a general theory and if students of the book are encouraged to have 'aha moments' of understanding, then this will lead to the concept of a textbook. If the book lacks a general theory, then the only option is to treat it as a holy book. But a book that contains a general theory can also be treated as a holy book. One sees this in systems of education that emphasize rote learning.

That brings us finally back to fundamentalism. Fundamentalism emerges in response to a new system of education with a different set of textbooks and holy books. When some society bases its education upon some holy book, then most of the members of that society simply assume that this book is the legitimate source of education. That is because a mental network functions silently under the surface as long as it is receiving consistent input. The concept of a holy book becomes explicit when a new and different system of education becomes dominant with its own set of holy books and textbooks. Fundamentalism then attempts to preserve the old system of education by packaging it within various religious books usually held together by some holy book. This response will be regarded by the new majority as fundamentalism and, as was pointed out earlier, the new majority will wonder why the old group is responding to the educational change with such emotional vigor. This interpretation of 'fundamentalism as extreme conservatism' can be seen in the research of Altemeyer (1992) who has emphasized the relationship between religious fundamentalism and right-wing authoritarianism. However, Williamson points out that "Fundamentalists sometimes may be militant, but largely when reacting to a perceived threat as posed by the larger culture to their absolute beliefs_-and even then, their resistance may take on forms that are nonviolent” (Williamson, 2010, p. 722). I suggest that the underlying problem is that the old system of education is taking a leap of faith to the TMN of some concept of God. Thus, when a new system of education becomes dominant, then the old system cannot defend itself by focusing upon content-because it is taking a leap of faith from limited content. The old group will then respond by focusing upon the leap of faith, which is done by explicitly regarding the textbooks of the old system as holy books.

It was mentioned earlier that a concept of God will become associated with various holy places, holy people, and holy rituals. This is a byproduct of taking a leap of faith. A TMN that is based in a truly universal theory will be triggered in all situations. But a TMN that is based in a leap of faith from some limited content will only be triggered in those areas of limited content. For instance, if a concept of God becomes mentally connected with helping those who are less fortunate than I am, then people will be conscious of God when helping those who are less fortunate than they are. Similarly, an old system of education will become mentally associated with previous eras when the holy books and textbooks of the old system of education formed the basis for the education of society, and anything that triggers MNs of this bygone era will cause members of the old society to become conscious of God. For instance, American Christian fundamentalism tends to associate 1950s American culture with Christianity because education acknowledged Christianity during this time and church membership was at a peak (Jones, 2019). Similarly, Jewish fundamentalism associates following God with wearing the clothing of $18^{\text {th }}$ century Eastern Europe when there was a major revival of Jewish religious fervor. 
Putting this all together, holy books and textbooks naturally accompany any system of education. Belief in a holy book can be protected by avoiding questioning from executive function, while a textbook must encourage such questioning in order to avoid being treated as a holy book. The average person becomes consciously aware that certain books are being treated as holy when a new system of education becomes dominant with a different set of authoritative texts. Members of the once-dominant group will then package the old system of education into a collection of authoritative texts. The old group will then be driven by its TMNs to behave in a manner that is consistent with its TMNs, recreate aspects of the previous era when these TMNs were accepted by the larger society, and avoid behavior of the new group that violates its TMNs. This will express itself as a conservative society that adheres to previous moral standards while rejecting new standards of morality. The members of the new dominant group will regard this reactionary response as religious fundamentalism.

What happens next will vary. A fundamentalist group may withdraw from larger society in order to apply the content of the holy book within some segregated community. This option can be seen in the Amish. A group may use political means to attempt to reimpose the previous system of education upon the larger society. This option can be seen in American right-wing politics. A group may also use military means to force the previous system upon society. This option can be seen in the 2021 takeover of Afghanistan by the Taliban. Or a group may show to the larger society that its system of education leads to better results than the new system. This happened with the fall of communism in the 1980s when it became obvious that communism with its system of education produced inferior results compared to Western society.

That brings us finally to the connection between religious fundamentalism and right-wing authoritarianism (Altemeyer, 1992). Altemeyer says that right-wing authoritarianism is based upon the three fundamental characteristics of authoritarian aggression, authoritarian submission, and conventionalism. Looking at these three traits more carefully, “The 'authoritarian submission' dimension can be defined as expressing attitudes favouring uncritical, respectful, obedient, submissive support for existing societal or group authorities and institutions” (Duckitt, 2010, p. 690). This describes an emotional hierarchy in which MNs that represent self and others are placed below MNs that represent leaders and societal institutions. “The 'authoritarian aggression' dimension can be defined as expressing attitudinal beliefs favouring the use of strict, tough, harsh, punitive, coercive social control” (p. 690). This describes MNs of authority imposing their structure upon MNs of self and others. And conventionalism "expresses the value and motivational goal of maintaining traditional lifestyles, norms, and morality” (p. 691). This describes a desire to preserve past MNs of culture and societal norms. Thus, right-wing authoritarianism relates to the second and third responses of the previous paragraph in which a once-dominant group uses either political or military means to reimpose its system of education upon the larger society_-but not to the first and fourth responses. Right-wing authoritarianism is consistent with the fundamentalist assumption that absolute truth is revealed to society through some authoritative source. Thus, there is a link between religious fundamentalism and right-wing authoritarianism. However, as Williamson points out, "Not all fundamentalists are militant, thus questioning the usefulness of such stereotypes” (Williamson, 2010, p. 722). 
Turning now to neurology, there is a relationship between fundamentalism and vmPFC activity. A neurological study of Vietnam veterans with brain damage summarized that "Fundamentalist religious beliefs arise from the integrated processing and computations in a distributed brain network, with the vmPFC as an essential hub in the circuitry" (Zhong, 2017, p. 20). Looking at this in more detail, the study found that "Participants with vmPFC lesions reported greater fundamentalism" (p. 18). The statement that the vmPFC is an essential hub of fundamentalism may seem to contradict the finding that vmPFC lesions lead to greater fundamentalism, but I suggest that two different aspects of fundamentalism are being described. On the one hand, fundamentalism is an attempt to avoid triggering the mental networks of the larger society, which suggests that vmPFC activity is being suppressed. But on the other hand, fundamentalism is a battle over mental networks, which means that the vmPFC is an essential hub. Fundamentalism is attempting to maintain its own mental networks, which it does by

gathering together frequently with fellow-minded believers in order to provide these mental networks with consistent input. These meetings are characterized by emotional fervor, indicating that mental networks are being activated.

Going further, the study found that "Greater relative dlPFC lesion damage was associated with greater reductions in cognitive flexibility and openness, and that less cognitive flexibility and openness in turn predicts an increase in fundamentalist beliefs” (p. 20). We saw earlier that physics students use the dorsolateral prefrontal to question the validity of naïve MNs. Fundamentalism suppresses this type of error checking, leading to cognitive inflexibility. This combination of cognitive inflexibility and gathering together with like-minded individuals to reinforce mental networks can be seen in the echo chamber (Cinelli, 2021). Fundamentalism may use a form of error-checking, but it will tend to be cognitively inflexible, characterized by repetition and quoting from approved texts.

\section{Nature}

The previous section suggested that a science textbook is similar to a holy book. Comparing these two may seem like comparing apples and oranges because holy books talk about God while science textbooks do not. However, a closer look indicates that science textbooks do talk about God. This God is commonly referred to as Nature with a capital ' $N$ ', and the attributes that are ascribed to Nature are attributes that could only be possessed by a divine being.

It would be interesting to compare descriptions of Nature with the traits discussed in a previous section, but this paper will limit itself to a single quote. "The overwhelming consensus of scientific opinion is that [biological organisms] were not designed and built by a Creator (a mental construct necessary to inject a human sense of purpose into existence) with purposes in mind for them. Instead, we believe (I'll put that as strongly as I can) they are products of Darwinian evolution... Bizarrely, evolutionists lead the world in substituting teleology for objectivity. How so? One major reason is the manner in which natural selection slipped seamlessly into the place of the Creator: the Natural Selector as the acceptable new face of the Great Designer” (Hanke, 2004, p. 147). Thus, interpreting the order-withincomplexity of nature as a personal being is being regarded as 'a mental construct' that is incompatible with scientific thought. However, this 'mental construct' is proving to be more powerful than scientific 
questioning because 'natural selection [has] slipped seamlessly into the place of the Creator'. Stated cognitively, scientific theory has formed a TMN that is mentally behaving like a divine being and, like any mental network, it is imposing its structure upon the mind leading to the concept of 'design' through 'natural selection'. Notice that the author is responding emotionally, implying the presence of theoretical emotion. Notice also that 'design' relates the attribute that 'God has a plan for my life' while 'natural selection' relates to the attribute that 'God can be seen in the interaction of personal events'.

Looking at this more generally, when scientific minds treat Nature as a God despite the strongest attempts not to do so, this provides strong evidence that one is dealing with a cognitive mechanism. It was suggested earlier that a concept of God emerges when a sufficiently general theory turns into a TMN and is interpreted personally. We see here that this progression will happen even when an entire group of scientifically trained people attempt to prevent it from happening. Saying this another way, religion is often viewed as a social construct, but in this case, a concept of deity is emerging despite the best social efforts to construct something else.

This also suggests that it is valid to treat the textbook as similar to a holy book. When the theories of a textbook become sufficiently general and are interpreted personally, then a concept of God will emerge and people will start talking as if some deity is guiding events. When this happens, then one has entered the realm of a holy book. However, because mental networks tend to function silently under the surface as long as they continue to receive consistent input, most members of a society will view the textbooksturned-holy-books of that society as merely the 'proper' form of education.

\section{Conspiracy Theories}

Fundamentalism was interpreted as a reaction by some societal group to the introduction of a new and different system of education. Fundamentalism contains an inherent leap of faith because a leap is being made from the specific words of some book to a TMN that expresses universal theological and moral statements.

Conspiracy theories also exhibit the characteristics of a TMN: "Just as scholars across disciplines have suggested that anthropomorphic thinking fuels conspiracy theories, scholars in political science, sociology, and literary studies have argued that conspiracy theories are a response to the apparent randomness of socio-political events. Social psychologists have shown that people are averse to this randomness, and impose meaning on it cognitively by perceiving patterns in events, including causal connections, even when none exist” (Douglas, 2018, p. 260). 'Anthropomorphic thinking' describes being motivated by MNs to interpret events as being caused by agents, and the paper refers specifically to the 'agency detector device' which we saw earlier when looking at the cognitive science of religion. Going further, 'perceiving patterns even when none exist' describes being motivated by theoretical emotion. The combination of anthropomorphic thinking and a drive to perceive order-withincomplexity describes the behavior of a TMN.

Looking further, conspiracy thinking satisfies an emotional need for order-within-complexity. "Systems of belief serve a purpose in guiding emotional and behavioural responses, and their 'gestalt' or overall 
consistency may be more important at a given time than consistency between peripheral beliefs in the system" (Douglas, 2018, p. 265). Notice that error-checking from executive function is being suppressed. And the desire for a universal theory can be seen in the finding that "One of the most common findings in the literature is that if a person believes in one conspiracy theory then they are more likely to believe in others” (p. 264). When a universal theory is being formulated by extrapolating from limited data in a manner that avoids error-checking, then this describes a leap of faith.

A cognitive explanation for conspiracy thinking becomes apparent if one recognizes that theories are like equations with variables that can be filled in with many different specific situations. Thus, if taking a leap of faith is treated as a general theory, then this theory remains valid as long as some segment of society takes a major leap of faith. Who precisely takes the leap is of secondary importance. This leads the following hypothesis: 1) a person makes a leap of faith from partial information to sweeping statements; 2) sufficient study occurs for this ideological leap to form a TMN; 3) some crisis occurs in which MNs of culture and identity as well as the TMN of the leap feel threatened by large forces. 4) the TMN of the ideological leap becomes preserved by applying it to 'them' rather than to 'us'. (In some cases, step 3 may happen before steps 1 and 2.)

These characteristics can be seen in the paper by Douglas. There is an ideological leap to a sweeping statement: "The idea that people in power (e.g., governments) cover up their actions to deceive the public is central to most conspiracy theories" (Douglas, 2018, p. 265). There is a crisis: "Conspiracy theories... tend to reduce engagement in important social domains and are associated with feelings of powerlessness, disillusionment and mistrust” (p. 280). In response to this crisis, a mindset used by 'us' is being applied to 'them'. There is "evidence that belief in conspiracy theories results from normal social cognitive processes - in this case the projection of one's own moral tendencies onto others" (p. 267). And this sweeping statement about 'them' transcends existing facts: “Conspiracy theories appear to be defined not by adherence to a particular alternative account, but by opposition to the official story and a belief that deception is taking place” (p. 266). This provides a possible reason why a conspiracy theory is so resistant to facts. What is being protected is not some theory but rather the TMN of leaping beyond the facts to discover the ultimate theory.

Notice that the conspiracy theory provides an alternative to the response of fundamentalism and may accompany a mindset of fundamentalism. Fundamentalism attempts to maintain some system of beliefs that is being threatened by the beliefs of the larger society through a leap of faith. A conspiracy theory applies a leap of faith to the behavior and beliefs of the larger society. In both cases the TMN of a leap of faith is being preserved.

Looking at a current example of a conspiracy theory, there is "strong support for the theory that COVID-19 CTs [conspiracy theories] form a monological belief system. Not only are the CTs highly correlated, but a large majority believe more than one. Moreover, mutually contradictory CTs are positively related to one another, even when standard predictors of CT beliefs are taken into account" (Miller, 2020, p. 325). On the one hand, the conspiracy theory is providing order-within-complexity. But on the other hand, this order is being constructed using facts that contradict one another. The only 
way to construct a theory out of contradictory facts is to take a leap of faith from the facts to the theory, in which case the contradictions actually encourage the taking of a leap.

This may explain why rational people are embracing conspiracy theories about Covid-19. A leap of faith can be maintained in modern society if rational thinking is limited to technical specializations and if these 'islands' of rational thought are integrated in a manner that is invisible to the average person. For instance, the typical modern person works within some specialized job while purchasing most necessities of life from a store. Such a person uses rational thought within the specialized job but has no idea how most items in a store are produced or how they travel from producer to store shelf. However, governments are responding to the Covid-19 crisis by instituting national structure in a highly visible manner, such as imposing national lockdowns, requiring everyone to wear masks, and instituting national vaccine campaigns. It was mentioned earlier that a sweeping statement cannot survive contact with facts. The coordinated response of governments to Covid-19 prevents the mind from making a leap of faith because it fills in the gap between specific occupation and global economy with highly visible, humanly organized behavior. I should emphasize that it is not conspiracy thinking to question if a government is capable of carrying out such a plan or to criticize some government for responding inadequately or incompetently. Conspiracy thinking goes beyond these visible facts to hypothesize some hidden agenda, and this search for a hidden agenda involves a leap of faith. Going further, governments do occasionally have hidden agendas, but conspiracy thinking goes beyond examining facts and understanding underlying motivations to obsessing over secret cabals and hidden agendas. This obsession indicates the presence of a TMN.

Looking at this from the other side, when the primary response of science is to debunk naïve MNs and suppress TMNs of deity, then preserving culture and religion will be equated with maintaining a leap of faith despite the skepticism of science. In the words of one author, "The decline of traditional religion in the West has been matched by a rise in the visibility of conspiracy theories. Conspiracy theories seem to fulfil some of the psychological needs addressed by religion, such as imposing a sense of order and agency upon the seemingly random and capricious world... These patterns have led some scholars to question whether conspiracy theories are, in some sense, a replacement for religious belief in an increasingly secular society” (Wood, 2018, p. 19).

In a related vein, the 'rapture doctrine' of evangelical Christianity has the characteristics of a conspiracy theory. This is the belief that Jesus will return from heaven to rescue Christians from an apostate world. This 'rapture' will be followed by a 'great tribulation' in which God rains destruction upon a satanic 'kingdom of the beast' (Lindsey, 1970). The rapture doctrine contains rational content because biblical passages and historical events are being studied. However, there is a literal 'leap of faith', because it is believed that the faithful will physically leap from secular Earth to the heaven of God. This physical leap mirrors the manner in which theology cognitively leaps from rational content to the TMN of a concept of God. This is another example of the mindset of the scholar imposing itself upon the data that is being studied.

The rapture doctrine qualifies as a conspiracy theory because most of the factual content is being ascribed to the governmental system of 'the antichrist'. This focus can be seen in the Left Behind books 
(LaHaye, 2011), a series of sixteen novels that focus upon the machinations of the antichrist and his 'kingdom of the beast'. These novels are obviously resonating with a widespread mindset because over 80 million copies have been sold.

The cognitive requirement for maintaining a leap of faith also provides a possible explanation for the tendency of evangelical Christians to reject the concept of global warming. Connecting this with the previous paragraph, "Believers in Christian end-times theology are less likely to support policies designed to curb global warming than are other Americans” (Barker, 2013, p. 1). Looking at this cognitively, the idea that humans should organize on a global scale to protect the planet violates the assumption of a leap of faith because it fills in the gap between local activity and global order with rational human activity. Instead, the 'true believer' uses rational thought locally and then takes a leap of faith to believing that God will step in to address global problems in some miraculous manner through the 'rapture'. The distinction between local human action and a global leap of faith can be seen in the following quote. "Evangelicals and other traditionalistic Christians are no less likely than other Americans to view environmental degradation as a problem... and are sometimes inclined to support private acts of environmental stewardship, such as recycling... [but] such Christians are particularly unlikely to support politically oriented environmental protection-especially when they hold inerrant views toward the Bible” (p. 2).

However, there is another side to the story. Mental networks add meaning to human existence. Objective scientific thought avoids subjective MNs as well as suppressing the TMN of Nature. Therefore, emotional meaning within modern scientific society can only be found by taking some leap of faith. Thus, it is inaccurate to regard those who take a leap of faith as ignorant or uneducated. Instead, a distinction needs to be made between a person taking a leap of faith that extends from that person's existing knowledge and expertise and a person taking a leap of faith that contradicts the knowledge and expertise of that person.

It should also be pointed out that conspiracy theories are not just a characteristic of right-wing Christian conservativism. While "It is likely that the left and the right differ in the type of conspiracy theories that they endorse... both extremes share a general proneness to conspiracy beliefs about societal events" (Van Prooijen, 2015, p. 7). This is consistent with the suggestion that conspiracy theories emerge from a combination of a leap of faith and societal marginalization. The right-wing fundamentalist feels marginalized in today's postmodern society, but similar feelings of marginalization may be felt by the left-wing individual who is taking a leap of faith to universal tolerance or a leap of faith to the concept of a 'living earth' (Lovelock, 2003).

\section{A Cognitive Meta-theory}

We have seen that TMNs can cause scholars to impose their mindsets upon the data that they are interpreting. More specifically, we saw that the mindset of objective science caused Thomas Kuhn, the discoverer of paradigms, to turn his back upon the concept of paradigms, as well as causing Semir Zeki, the discover of the neurological connection between beauty and the vmPFC, to deny historical concepts of beauty. And we have seen that objective science refers to Nature as a divine being-while 
at the same time denying that it is referring to Nature as a divine being and doing its best to squelch such language. Thus, attempting to avoid TMNs does not work. The alternative is to construct a general theory which explicitly acknowledges that science is emotionally guided by TMNs and which explicitly recognizes that sufficiently general theories will create concepts of God when applied personally, knowing that using this theory will cause it to turn into a TMN which will impose its structure upon the mind. This means recognizing at a theoretical level that science has religious overtones, and that this is not just a flaw to which science is susceptible, but rather an intrinsic — and inescapable-feature of scientific thought. However, this does not mean converting to some existing religion. That is because all existing religious thought performs some kind of leap of faith and a leap of faith, by its very nature, suppresses the careful thinking of scientific analysis. Thus, what is required is a reformulation of religion that avoids taking any leap of faith.

A cognitive meta-theory has been developed which appears to satisfy these requirements and this paper has described major elements of this theory. The theory was initially developed through an extensive analysis of biographies (Friesen, 1986), it provides a cognitive explanation for theology at the level of systematic theology as well as providing a cognitive explanation for scientific thought (Friesen, 2015, 2020), it maps in detail on to neurology (Friesen, 2019), it can be used to analyze many aspects of linguistics and social interaction (Friesen, 2013), it has been used to analyze Economics (Friesen, 2021a), and it has been applied to many other subjects in a less rigorous manner.

A meta-theory takes existing theories and places them within a more general package. It does this by looking beyond the terminology of a specific theory to examine the functions and concepts described by that theory. For instance, this paper has been quoting extensively from existing papers using existing terminology. However, the concepts that have been discussed are all aspects of the meta-theory of mental symmetry, which can also be described using the language of mental symmetry. This sort of mapping from one theory to another is only possible when everyone is examining the same subject material. This can be done when studying the physical world because every observer is subject to the same natural processes. It can also be done when studying the mind, because the same mental architecture will impose its structure upon everything that it examines. Thus, it is possible to construct a meta-theory of cognition by comparing how the mind is being used in different fields and specializations. This methodology was described in a previous article (Friesen, 2021c).

The imposition of mental architecture upon human thought goes beyond the realm of mental networks but interacts with mental networks. Science and technology attempt to cooperate with nature rather than fight nature by constructing and following mental networks that are consistent with natural processes. Similarly, it is possible to cooperate with the structure of the mind rather than fight the mind by constructing and following mental networks that are consistent with how the mind functions.

Notice that analogical reasoning is being used to construct a meta-theory of cognition, because one is looking for analogous thinking in different realms of thought and behavior. Analogical reasoning forms a fundamental aspect of normal human thought (Lakoff, 2008) and analogical thinking is compatible with scientific thought if it is done in a semi-rigorous manner, which includes looking beyond surface 
similarities to search for underlying functional parallels as well as mapping one topic systematically onto another (Gentner, 1993).

A cognitive meta-theory is one step removed from physical evidence. Objective science tends to view the physical world with its physical objects as the only valid topic of discussion. This is actually a contradiction in terms because following such a methodology will form a TMN which will mentally impose this interpretation upon physical evidence. This leads to the contradiction of being mentally driven by a TMN to deny that one is being driven by any sort of mental construct such as a TMN. A cognitive meta-theory that is one step removed from physical evidence recognizes the fundamental role that TMNs play in formulating scientific thought while at the same time recognizing that theories must be consistent with empirical evidence. Thus, instead of viewing physical reality as the only valid topic of discussion, physical evidence is treated as a constraint that guides rational discussion. This relates to the idea of constructing and following mental networks that are consistent with natural processes.

Moving one step away from physical evidence to cognition also makes it possible to study nonphysical topics in a rational manner. The meta-theory of mental symmetry was constructed by using analogical reasoning to compare different forms of thought. This methodology can be applied to forms of thought based in empirical evidence, but it can also be applied to topics such as religion that go beyond the physical to the non-physical and beyond the natural to the supernatural. This paper has illustrated what it means to apply the same form of analogical reasoning to both scientific and religious thought.

This type of cognitive analysis has also been used to analyze about half of the original Greek text of the Christian New Testament (as well as some passages of the Jewish Bible-which Christianity refers to as the 'Old Testament'). The general conclusion is that such a cognitive analysis works. One does not end up with nonsense but rather with significant cognitive principles that appear to be consistent with the functioning of the mind. Such an analysis sidesteps attempting to determine the empirical validity of the text and focuses instead upon cognitive principles. Another general conclusion is that a cognitive analysis fits the original Greek text better than the current theological interpretation with its leap of faith. These conclusions will be illustrated by examining one passage from the Gospel of Matthew.

Matthew 8 contains the story of Jesus healing the centurion's servant. The quoted passage describes the centurion asking Jesus to heal his servant.

Lord, I am not worthy for You to come under my roof, but just say the word, and my servant will be healed. For I also am a man under authority, with soldiers under me; and I say to this one, 'Go!' and he goes, and to another, 'Come!' and he comes, and to my slave, 'Do this!' and he does it.” Now when Jesus heard this, He was amazed and said to those who were following, "Truly I say to you, I have not found such great faith with anyone in Israel” (Matthew 8: 5 - 10, NASB).

Looking at this cognitively, the centurion was a secular, non-Jewish person. He redirects the attention from MNs of personal status to the words of abstract theory. He describes how the structure of his personal environment functions. He then suggests that the religious realm of God functions in a manner that is analogically similar. Jesus responds by saying that this form of analogical reasoning exhibits 
greater faith than any theological 'leap of faith' taken within the religious community. Consistent with this interpretation, the Greek word for faith (ríotıv) actually means to be persuaded, which suggests that the concept of a 'leap of faith' contradicts the meaning of the word 'faith'. Concluding, this story states that using analogical reasoning to move from the physical to the non-physical is superior to using theological reasoning to take a leap of faith, providing a theological justification for reformulating religion as a cognitive meta-theory.

As for aliens (and angels), I have found that the stories start making cognitive sense if one hypothesizes an exchange of concrete thought and abstract thought. Using the language of mental networks, this means that aliens acquire their TMNs from some sort of 'abstract' external environment, while having to use internal thought to generate the experiential MNs that humans acquire from the physical environment. This hypothesis sidesteps the demand for empirical evidence while providing a starting point for discussing the subject in a rational manner without having to make some leap of faith. It is

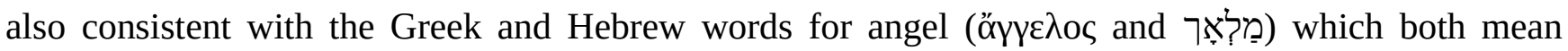
'messenger', implying beings who 'embody' abstract messages in some manner. And exchanging concrete thought and abstract thought involves the sort of symmetry that a physicist finds beautiful.

\section{Conclusion}

The mental network (MN) provides an integrated explanation for the diverse functions of the vmPFC. Concepts of self and others appear to be based in MNs while Theory of Mind combines various MNs to guess what is happening within the minds of others. The MN can be combined with the concept of theoretical emotion to come up with the cognitive mechanism of a TMN or theoretical mental network. The TMN adds an extra dimension to both concepts of self and Theory of Mind. It also provides an integrated explanation for many facets of high level human thought, including beauty, paradigms, education, ideologies, and conspiracy theories. And TMNs can be used to explain the formation of a concept of God as well as explain what motivates mysticism, theology, and fundamentalism. While neurological evidence linking TMNs with the vmPFC is limited, it does exist.

Placing this paper into the larger perspective, the cognitive meta-theory of mental symmetry suggests that the mind is composed of seven interacting cognitive modules. These modules can interact in one of three different ways: mental networks, the rigorous thinking of technical thought, and the analogies of normal thought. Each of these three modes of thought can be subdivided into a concrete and abstract component: Thus, there are experiential MNs and TMNs. There is concrete technical thought with its sense of cause-and-effect and there is abstract technical thought with its precise definitions. And there are the metaphors of concrete normal thought and the analogies of abstract normal thought. This paper has focused upon mental networks and their emotional impact while also referring to the other two forms of thinking.

\section{Acknowledgements}

I would like to thank Angelina Van Dyke for providing detailed feedback on this paper. 


\section{References}

Allaire-Duquette, G., Foisy, L. M. B., Potvin, P., Riopel, M., Larose, M., \& Masson, S. (2021). An fMRI study of scientists with a Ph. D. in physics confronted with naive ideas in science. NPJ science of learning, 6(1), 1-12.

Altemeyer, B., \& Hunsberger, B. (1992). Authoritarianism, religious fundamentalism, quest, and prejudice. The international journal for the psychology of religion, 2(2), 113-133.

Apperly, I. A. (2008). Beyond simulation-theory and theory-theory: why social cognitive neuroscience should use its own concepts to study "Theory of Mind”. Cognition, 107(1), 266283.

Barbey, A. K., Colom, R., \& Grafman, J. (2013). Dorsolateral prefrontal contributions to human intelligence. Neuropsychologia, 51(7), 1361-1369.

Barendse, M. E., Cosme, D., Flournoy, J. C., Vijayakumar, N., Cheng, T. W., Allen, N. B., \& Pfeifer, J. H. (2020). Neural correlates of self-evaluation in relation to age and pubertal development in early adolescent girls. Developmental cognitive neuroscience, 44, 100799.

Barker, D. C., \& Bearce, D. H. (2013). End-times theology, the shadow of the future, and public resistance to addressing global climate change. Political Research Quarterly, 66(2), 267-279.

Barrett, J. L., \& Keil, F. C. (1996). Conceptualizing a nonnatural entity: Anthropomorphism in God concepts. Cognitive psychology, 31(3), 219-247.

Barrett, J. L. (2007). Cognitive Science of Religion: What Is It and Why Is It? Religion Compass, 1(6), 768-786.

Barrier, R. (2016). What is the Difference between Praise and Worship? Retrieved from https://www.crosswalk.com/church/pastors-or-leadership/ask-roger/what-is-the-differencebetween-praise-and-worship.html

Bartra, O., McGuire, J. T., \& Kable, J. W. (2013). The valuation system: a coordinate-based metaanalysis of BOLD fMRI experiments examining neural correlates of subjective value. Neuroimage, 76, 412-427.

Belfi, A. M., Vessel, E. A., Brielmann, A., Isik, A. I., Chatterjee, A., Leder, H., ... \& Starr, G. G. (2019). Dynamics of aesthetic experience are reflected in the default-mode network. NeuroImage, 188, 584-597.

Benoit, R. G., Szpunar, K. K., \& Schacter, D. L. (2014). Ventromedial prefrontal cortex supports affective future simulation by integrating distributed knowledge. Proceedings of the National Academy of Sciences, 111(46), 16550-16555.

Berridge, K. C., \& Robinson, T. E. (2016). Liking, wanting, and the incentive-sensitization theory of addiction. American Psychologist, 71(8), 670.

Boes, A. D., Bechara, A., Tranel, D., Anderson, S. W., Richman, L., \& Nopoulos, P. (2009). Right ventromedial prefrontal cortex: a neuroanatomical correlate of impulse control in boys. Social cognitive and affective neuroscience, 4(1), 1-9. 
Bonnici, H. M., \& Maguire, E. A. (2018). Two years later-Revisiting autobiographical memory representations in vmPFC and hippocampus. Neuropsychologia, 110, 159-169.

Bosmans, G., Bakermans-Kranenburg, M. J., Vervliet, B., Verhees, M. W., \& van IJzendoorn, M. H. (2020). A learning theory of attachment: Unraveling the black box of attachment development. Neuroscience \& Biobehavioral Reviews, 113, 287-298.

Bowren, M. D., Croft, K. E., Reber, J., \& Tranel, D. (2018). Choosing spouses and houses: Impaired congruence between preference and choice following damage to the ventromedial prefrontal cortex. Neuropsychology, 32(3), 280.

Brod, G., \& Shing, Y. L. (2018). Specifying the role of the ventromedial prefrontal cortex in memory formation. Neuropsychologia, 111, 8-15.

Brod, G., \& Shing, Y. L. (2019). A boon and a bane: Comparing the effects of prior knowledge on memory across the lifespan. Developmental psychology, 55(6), 1326.

Broom, T. W., Chavez, R. S., \& Wagner, D. D. (2021). Becoming the King in the North: identification with fictional characters is associated with greater self-other neural overlap. Social cognitive and affective neuroscience, 16(6), 541-551.

Brown, V. M., Wilson, J., Hallquist, M. N., Szanto, K., \& Dombrovski, A. Y. (2020). Ventromedial prefrontal value signals and functional connectivity during decision-making in suicidal behavior and impulsivity. Neuropsychopharmacology, 45(6), 1034-1041.

Buber, M. (2012). I and Thou. eBookIt.com.

Bzdok, D., Langner, R., Schilbach, L., Engemann, D. A., Laird, A. R., Fox, P. T., \& Eickhoff, S. (2013). Segregation of the human medial prefrontal cortex in social cognition. Frontiers in human neuroscience, 7, 232.

Cameron, C. D., Reber, J., Spring, V. L., \& Tranel, D. (2018). Damage to the ventromedial prefrontal cortex is associated with impairments in both spontaneous and deliberative moral judgments. Neuropsychologia, 111, 261-268.

Cinelli, M., Morales, G. D. F., Galeazzi, A., Quattrociocchi, W., \& Starnini, M. (2021). The echo chamber effect on social media. Proceedings of the National Academy of Sciences, 118(9).

Cloutier, J., \& Gyurovski, I. (2014). Ventral medial prefrontal cortex and person evaluation: Forming impressions of others varying in financial and moral status. Neuroimage, 100, 535543.

Cohen-Zimerman, S., Cristofori, I., Zhong, W., Bulbulia, J., Krueger, F., Gordon, B., \& Grafman, J. (2020). Neural underpinning of a personal relationship with God and sense of control: A lesion-mapping study. Cognitive, Affective, \& Behavioral Neuroscience, 20(3), 575-587.

Corradi-Dell'Acqua, C., Hofstetter, C., \& Vuilleumier, P. (2014). Cognitive and affective theory of mind share the same local patterns of activity in posterior temporal but not medial prefrontal cortex. Social Cognitive and Affective Neuroscience, 9(8), 1175-1184.

Cristofori, I., Bulbulia, J., Shaver, J. H., Wilson, M., Krueger, F., \& Grafman, J. (2016). Neural correlates of mystical experience. Neuropsychologia, 80, 212-220. 
Dirac, P. A. M. (1963). The evolution of the physicist's picture of nature. Scientific American, 208(5), 45-53.

Doré, B. P., Scholz, C., Baek, E. C., Garcia, J. O., O’Donnell, M. B., Bassett, D. S., ... \& Falk, E. B. (2019). Brain activity tracks population information sharing by capturing consensus judgments of value. Cerebral Cortex, 29(7), 3102-3110.

Douglas, K. M., \& Sutton, R. M. (2018). Why conspiracy theories matter: A social psychological analysis. European Review of Social Psychology, 29(1), 256-298.

Dreyfus, S. E. (2004). The five-stage model of adult skill acquisition. Bulletin of science, technology \& society, 24(3), 177-181.

Duckitt, J., Bizumic, B., Krauss, S. W., \& Heled, E. (2010). A tripartite approach to right-wing authoritarianism: The authoritarianism-conservatism-traditionalism model. Political Psychology, 31(5), 685-715.

Erdeniz, B., \& Done, J. (2019). Common and Distinct Functional Brain Networks for Intuitive and Deliberate Decision Making. Brain sciences, 9(7), 174.

Farovik, A., Place, R. J., McKenzie, S., Porter, B., Munro, C. E., \& Eichenbaum, H. (2015). Orbitofrontal cortex encodes memories within value-based schemas and represents contexts that guide memory retrieval. Journal of Neuroscience, 35(21), 8333-8344.

Ferguson, M. A., Nielsen, J. A., King, J. B., Dai, L., Giangrasso, D. M., Holman, R., ... \& Anderson, J. S. (2018). Reward, salience, and attentional networks are activated by religious experience in devout Mormons. Social neuroscience, 13(1), 104-116.

Friesen, E. L., (1986). Cognitive Styles in History: Contributor and Server. Victoria, BC: Lane Friesen, Inc.

Friesen, E. L., (1986). Cognitive Styles in History: Perceiver and Mercy. Victoria, BC: Lane Friesen, Inc.

Friesen, L. A., \& Van Dyke, A. (2013). A Cognitive Meta-Theory for TESOL. Retrieved from researchgate.net. doi: 10.13140/RG.2.1.3552.2721

Friesen, L. A., (2015). Natural Cognitive Theology. Abbotsford, BC: Lorin Friesen.

Friesen, L. A., (2019). Mapping a cognitive theory onto neurology. Retrieved from researchgate.net. doi: 10.13140/RG.2.2.28982.24641

Friesen, L. A., (2020). A cognitive model of science and theology. Retrieved from researchgate.net. doi: 10.13140/RG.2.2.27653.17122/1

Friesen, L. A., (2021a). A cognitive model of economics. Retrieved from researchgate.net. doi: 10.13140/RG.2.2.17044.83847

Friesen, L. A., (2021b). Theories generate emotions. Academia Letters, Article 2682.

Friesen, L. A., (2021c). Using mental bias to construct a model of cognition. Academia Letters, Article 1681. 
Gentner, D., \& Jeziorski, M. 1993. The shift from metaphor to analogy in Western science. In A. Ortony (Ed.), Metaphor and thought: 447-480. Cambridge: Cambridge University Press.

Gherman, S., \& Philiastides, M. G. (2018). Human VMPFC encodes early signatures of confidence in perceptual decisions. Elife, 7, e38293.

Gilboa, A., \& Marlatte, H. (2017). Neurobiology of schemas and schema-mediated memory. Trends in cognitive sciences, 21(8), 618-631.

Ginty, A. T., Kraynak, T. E., Kuan, D. C., \& Gianaros, P. J. (2019). Ventromedial prefrontal cortex connectivity during and after psychological stress in women. Psychophysiology, 56(11), e13445.

Gobet, F., \& Chassy, P. (2008). Towards an alternative to Benner's theory of expert intuition in nursing: a discussion paper. International journal of nursing studies, 45(1), 129-139.

Gonzalez, S. T., \& Fanselow, M. S. (2020). The role of the ventromedial prefrontal cortex and context in regulating fear learning and extinction (Vol. 13, No. 3, p. 459). Educational Publishing Foundation.

Gopnik, A. (1996). The scientist as child. Philosophy of science, 63(4), 485-514.

Graybiel, A. M., \& Smith, K. S. (2014). Good habits, bad habits. Scientific American, 310(6), 3843.

Grice, H. (1975). In Syntax and Semantics, P. Cole, J. L. Morgan, Eds. Academic Press, 3, 41-58.

Guan, F., Zhao, S., Chen, S., Lu, S., Chen, J., \& Xiang, Y. (2019). The neural correlate difference between positive and negative awe. Frontiers in human neuroscience, 13, 206.

Hanke, D., 2004. Teleology: The explanation that bedevils biology. In: Explanations: Styles of explanation in science. New York: Oxford University Press, pp. 143-155.

Hebscher, M., \& Gilboa, A. (2016). A boost of confidence: The role of the ventromedial prefrontal cortex in memory, decision-making, and schemas. Neuropsychologia, 90, 46-58.

Heisenberg, W. (1971). Physics and Beyond: Encounters and Conversations.

Ishizu, T., \& Zeki, S. (2011). Toward a brain-based theory of beauty. PloS one, 6(7), e21852.

Ito, A., Yoshida, K., Takeda, K., Sawamura, D., Murakami, Y., Hasegawa, A., ... \& Izuma, K. (2020). The role of the ventromedial prefrontal cortex in automatic formation of impression and reflected impression. Human brain mapping, 41(11), 3045-3058.

Jacobs, A. (2017). Wokeness and myth on campus. The New Atlantis, 33-44.

Jones, J. M. (2019). US church membership down sharply in past two decades. Gallup, Politics, April, 18.

Kawabata, H., \& Zeki, S. (2004). Neural correlates of beauty. Journal of neurophysiology, 91(4), 1699-1705.

Koenigs, M., Huey, E. D., Raymont, V., Cheon, B., Solomon, J., Wassermann, E. M., \& Grafman, J. (2008). Focal brain damage protects against post-traumatic stress disorder in combat veterans. Nature neuroscience, 11(2), 232-237. 
Kolling, N., Behrens, T. E., Wittmann, M. K., \& Rushworth, M. F. (2016). Multiple signals in anterior cingulate cortex. Current opinion in neurobiology, 37, 36-43.

Kolling, N., Braunsdorf, M., Vijayakumar, S., Bekkering, H., Toni, I., \& Mars, R. B. (2021). Constructing others' beliefs from one's own using medial frontal cortex. Journal of Neuroscience.

Konova, A. B., \& Goldstein, R. Z. (2019). The emerging neuroscience of appetitive and drug cue extinction in humans. Psychopharmacology, 236(1), 407-414.

Kuhn, T. (1970) The structure of scientific revolutions. Second Edition. University of Chicago press.

Kühn, S., Müller, B. C., Van Baaren, R. B., Wietzker, A., Dijksterhuis, A., \& Brass, M. (2010). Why do I like you when you behave like me? Neural mechanisms mediating positive consequences of observing someone being imitated. Social Neuroscience, 5(4), 384-392.

Kuntoro, I. A., Saraswati, L., Peterson, C., \& Slaughter, V. (2013). Micro-cultural influences on theory of mind development: A comparative study of middle-class and pemulung children in Jakarta, Indonesia. International Journal of Behavioral Development, 37(3), 266-273.

LaHaye, T., \& Jenkins, J. B. (2011). Left behind: A novel of the earth's last days. Tyndale House Publishers, Inc.

Lakoff, G., \& Johnson, M. (2008). Metaphors we live by. University of Chicago press.

Lieberman, M. D., Straccia, M. A., Meyer, M. L., Du, M., \& Tan, K. M. (2019). Social, self, (situational), and affective processes in medial prefrontal cortex (MPFC): Causal, multivariate, and reverse inference evidence. Neuroscience \& Biobehavioral Reviews, 99, 311-328.

Liljedahl, P. G. (2005). Mathematical discovery and affect: The effect of AHA! experiences on undergraduate mathematics students. International Journal of Mathematical Education in Science and Technology, 36(2-3), 219-234.

Lin, W. J., Horner, A. J., \& Burgess, N. (2016). Ventromedial prefrontal cortex, adding value to autobiographical memories. Scientific reports, 6(1), 1-10.

Lindsey, H., \& Carlson, C. C. (1970). The late great planet earth. Zondervan.

Lovelock, J. (2003). Gaia: the living Earth. Nature, 426(6968), 769-770.

Luo, Y., Eickhoff, S. B., Hetu, S., \& Feng, C. (2018). Social comparison in the brain: A coordinatebased meta-analysis of functional brain imaging studies on the downward and upward comparisons. Human brain mapping, 39(1), 440-458.

Luttenberger, S., Wimmer, S., \& Paechter, M. (2018). Spotlight on math anxiety. Psychology research and behavior management, $11,311$.

Lutzker, M. A. (1982). Max Weber and the analysis of modern bureaucratic organization: Notes toward a theory of appraisal. The American Archivist, 45(2), 119-130.

Manohar, S., Lockwood, P., Drew, D., Fallon, S. J., Chong, T. T., Jeyaretna, D. S., ... \& Husain, M. (2021). Reduced decision bias and more rational decision making following ventromedial prefrontal cortex damage. cortex, 138, 24-37. 
Manuel, A. L., Murray, N. W., \& Piguet, O. (2019). Transcranial direct current stimulation (tDCS) over vmPFC modulates interactions between reward and emotion in delay discounting. Scientific Reports, 9(1), 1-9.

McKinnon, A. (1993). Kierkegaard and “The Leap of Faith”. Kierkegaardiana, 16.

McLeod, S. (2010). Formal Operational Stage. Simply Psychology.

McLeod, S. (2018). The Preoperational Stage of Cognitive Development. Simply Psychology.

McCloskey, M. (1983). Intuitive Physics. Scientific American, 248(4), 122-130. https://doi.org/10.1038/SCIENTIFICAMERICAN0483-122

McCormick, C., Ciaramelli, E., De Luca, F., \& Maguire, E. A. (2018). Comparing and contrasting the cognitive effects of hippocampal and ventromedial prefrontal cortex damage: a review of human lesion studies. Neuroscience, 374, 295-318.

Mehta, P. S., Tu, J. C., LoConte, G. A., Pesce, M. C., \& Hayden, B. Y. (2019). Ventromedial prefrontal cortex tracks multiple environmental variables during search. Journal of Neuroscience, 39(27), 5336-5350.

Mi, Q., Wang, C., Camerer, C. F., \& Zhu, L. (2021). Reading between the lines: Listener's vmPFC simulates speaker cooperative choices in communication games. Science Advances, 7(10), eabe6276.

Miller, B. W., Wroten, M. G., Sacramento, A. D., Silva, H. E., Shin, C. B., Vieira, P. A., ... \& Szumlinski, K. K. (2017). Cocaine craving during protracted withdrawal requires PKCE priming within vmPFC. Addiction biology, 22(3), 629-639.

Miller, J. M. (2020). Do COVID-19 conspiracy theory beliefs form a monological belief system?. Canadian Journal of Political Science/Revue canadienne de science politique, 53(2), 319-326.

Mitchell, J. P., Macrae, C. N., \& Banaji, M. R. (2006). Dissociable medial prefrontal contributions to judgments of similar and dissimilar others. Neuron, 50(4), 655-663.

Montano, U. (2013). Explaining beauty in mathematics: An aesthetic theory of mathematics (Vol. 370). Springer Science \& Business Media.

Murphy, A. R. (1997). Tolerance, toleration, and the liberal tradition. Polity, 29(4), 593-623.

Norman, G. R., Monteiro, S. D., Sherbino, J., Ilgen, J. S., Schmidt, H. G., \& Mamede, S. (2017). The causes of errors in clinical reasoning: cognitive biases, knowledge deficits, and dual process thinking. Academic Medicine, 92(1), 23-30.

Padoa-Schioppa, C., \& Conen, K. E. (2017). Orbitofrontal cortex: a neural circuit for economic decisions. Neuron, 96(4), 736-754.

Patterson, T. K., \& Knowlton, B. J. (2018). Subregional specificity in human striatal habit learning: a meta-analytic review of the fMRI literature. Current Opinion in Behavioral Sciences, 20, 75-82.

Pelletier, G., \& Fellows, L. K. (2019). A critical role for human ventromedial frontal lobe in value comparison of complex objects based on attribute configuration. Journal of Neuroscience, 39(21), 4124-4132. 
Pennington, Z. T., Anderson, A. S., \& Fanselow, M. S. (2017). The ventromedial prefrontal cortex in a model of traumatic stress: fear inhibition or contextual processing?. Learning \& Memory, 24(9), 400-406.

Pretus, C., Hamid, N., Sheikh, H., Gómez, Á., Ginges, J., Tobeña, A., ... \& Atran, S. (2019). Ventromedial and dorsolateral prefrontal interactions underlie will to fight and die for a cause. Social cognitive and affective neuroscience, 14(6), 569-577.

Riforgiato, L. R. (1972). The Unified Thought of Jonathan Edwards. Thought: Fordham University Quarterly, 47(4), 599-610.

Robins, L. N. (1993). Vietnam veterans’ rapid recovery from heroin addiction: A fluke or normal expectation?. Addiction, 88(8), 1041-1054.

Rolls, E. T., Cheng, W., \& Feng, J. (2020). The orbitofrontal cortex: reward, emotion and depression. Brain Communications, 2(2), fcaa196.

Ruffman, T. (2014). To belief or not belief: Children's theory of mind. Developmental review, 34(3), 265-293.

Schacter, D. L., Benoit, R. G., \& Szpunar, K. K. (2017). Episodic future thinking: Mechanisms and functions. Current opinion in behavioral sciences, 17, 41-50.

Shamay-Tsoory, S. G., \& Aharon-Peretz, J. (2007). Dissociable prefrontal networks for cognitive and affective theory of mind: a lesion study. Neuropsychologia, 45(13), 3054-3067.

Shan, Y. (2020). Kuhn’s “wrong turning” and legacy today. Synthese, 197(1), 381-406.

Sloman, S. A. (1996). The empirical case for two systems of reasoning. Psychological bulletin, 119(1), 3.

Spalding, K. N., Schlichting, M. L., Zeithamova, D., Preston, A. R., Tranel, D., Duff, M. C., \& Warren, D. E. (2018). Ventromedial prefrontal cortex is necessary for normal associative inference and memory integration. Journal of Neuroscience, 38(15), 3767-3775.

Stendardi, D., Biscotto, F., Bertossi, E., \& Ciaramelli, E. (2021). Present and future self in memory: the role of vmPFC in the self-reference effect. Social Cognitive and Affective Neuroscience.

Streib, H., Klein, C., Keller, B., \& Hood, R. (2020). The Mysticism Scale as Measure for Subjective Spirituality.

Sui, J., \& Gu, X. (2017). Self as object: Emerging trends in self research. Trends in Neurosciences, 40(11), 643-653.

Tversky, A., \& Kahneman, D. (1992). Advances in prospect theory: Cumulative representation of uncertainty. Journal of Risk and uncertainty, 5(4), 297-323.

Van Dijk, T. A. (2006). Ideology and discourse analysis. Journal of political ideologies, 11(2), 115140.

Van Elk, M., Arciniegas Gomez, M. A., van der Zwaag, W., Van Schie, H. T., \& Sauter, D. (2019). The neural correlates of the awe experience: Reduced default mode network activity during feelings of awe. Human brain mapping, 40(12), 3561-3574. 
Van Prooijen, J. W., Krouwel, A. P., \& Pollet, T. V. (2015). Political extremism predicts belief in conspiracy theories. Social Psychological and Personality Science, 6(5), 570-578.

Vazire, S., \& Mehl, M. R. (2008). Knowing me, knowing you: the accuracy and unique predictive validity of self-ratings and other-ratings of daily behavior. Journal of personality and social psychology, 95(5), 1202.

Vessel, E. A., Isik, A. I., Belfi, A. M., Stahl, J. L., \& Starr, G. G. (2019). The default-mode network represents aesthetic appeal that generalizes across visual domains. Proceedings of the National Academy of Sciences, 116(38), 19155-19164.

Vetter, N. C., Weigelt, S., Döhnel, K., Smolka, M. N., \& Kliegel, M. (2014). Ongoing neural development of affective theory of mind in adolescence. Social Cognitive and Affective Neuroscience, 9(7), 1022-1029.

Williamson, W., Hood Jr, R. W., Ahmad, A., Sadiq, M., \& Hill, P. C. (2010). The Intratextual Fundamentalism Scale: Cross-cultural application, validity evidence, and relationship with religious orientation and the Big 5 factor markers. Mental Health, Religion \& Culture, 13(7-8), 721-747.

Wood, M., \& Douglas, K. (2018). Are conspiracy theories a surrogate for God?. In Handbook of conspiracy theory and contemporary religion (pp. 87-105). Brill.

Woods, R., \& Walrath, B. (Eds.). (2007). The message in the music: Studying contemporary praise and worship. Abingdon Press.

Wulff, D. M. (2014). Mystical experiences. In E. Cardeña, S. J. Lynn, \& S. Krippner (Eds.), Varieties of anomalous experience: Examining the scientific evidence (pp. 369-408). American Psychological Association.

Yaden, D. B., Eichstaedt, J. C., Schwartz, H. A., Kern, M. L., Le Nguyen, K. D., Wintering, N. A., ... \& Newberg, A. B. (2016). The language of ineffability: Linguistic analysis of mystical experiences. Psychology of Religion and Spirituality, 8(3), 244.

Young, C. B., Wu, S. S., \& Menon, V. (2012). The neurodevelopmental basis of math anxiety. Psychological science, 23(5), 492-501.

Zeki, S., Romaya, J. P., Benincasa, D. M., \& Atiyah, M. F. (2014). The experience of mathematical beauty and its neural correlates. Frontiers in human neuroscience, 8, 68.

Zeki, S., Chén, O. Y., \& Romaya, J. P. (2018). The biological basis of mathematical beauty. Frontiers in human neuroscience, 12, 467.

Zeki, S. (2019). Notes towards a (neurobiological) definition of beauty. Gestalt Theory, 41(2), 107-112.

Zhong, W., Cristofori, I., Bulbulia, J., Krueger, F., \& Grafman, J. (2017). Biological and cognitive underpinnings of religious fundamentalism. Neuropsychologia, 100, 18-25.

Note: When the official version of the paper was behind a pay wall, then the quotes were taken from a public version of the paper and the page number of the quote was estimated. 